\title{
DEGENERACY AND FINITENESS THEOREMS FOR MEROMORPHIC MAPPINGS IN SEVERAL COMPLEX VARIABLES
}

\author{
SI DUC QUANG
}

\begin{abstract}
In this article, we prove that there are at most two meromorphic mappings of $\mathbf{C}^{m}$ into $\mathbf{P}^{n}(\mathbf{C})(n \geqslant 2)$ sharing $2 n+2$ hyperplanes in general position regardless of multiplicity, where all zeros with multiplicities more than certain values do not need to be counted. We also show that if three meromorphic mappings $f^{1}, f^{2}, f^{3}$ of $\mathbf{C}^{m}$ into $\mathbf{P}^{n}(\mathbf{C})(n \geqslant 5)$ share $2 n+1$ hyperplanes in general position with truncated multiplicity then the map $f^{1} \times f^{2} \times f^{3}$ is linearly degenerate.
\end{abstract}

\section{INTRODUCTION}

In 1926, R. Nevanlinna 3 showed that two distinct nonconstant meromorphic functions $f$ and $g$ on the complex plane $\mathbf{C}$ cannot have the same inverse images for five distinct values, and that $g$ is a special type of linear fractional transformation of $f$ if they have the same inverse images counted with multiplicities for four distinct values [3]. These results are usually called the five values and the values theorems of R. Nevanlinna.

After that, many authors extended and improved the results of Nevanlinna to the case of meromorphic mappings into complex projective sapces. The extensions of the five values theorem are usually called the uniqueness theorems, and the extensions of the four values theorem are usually called the finiteness theorems. Here we formulate some recent results on this problem.

To state some of them, first of all we recall the following.

Let $f$ be a nonconstant meromorphic mapping of $\mathbf{C}^{m}$ into $\mathbf{P}^{n}(\mathbf{C})$ and $H$ a hyperplane in $\mathbf{P}^{n}(\mathbf{C})$. Let $k$ be a positive integer or $k=\infty$. Denote by $\nu_{(f, H)}$ the map of $\mathbf{C}^{m}$ into $\mathbf{Z}$ whose value $\nu_{(f, H)}(a)\left(a \in \mathbf{C}^{m}\right)$ is the intersection multiplicity of the images of $f$ and $H$ at $f(a)$. For every $z \in \mathbf{C}^{m}$, we set

$$
\begin{array}{r}
\nu_{(f, H), \leqslant k}(z)= \begin{cases}0 & \text { if } \nu_{(f, H)}(z)>k, \\
\nu_{(f, H)}(z) & \text { if } \nu_{(f, H)}(z) \leqslant k,\end{cases} \\
\text { and } \nu_{(f, H), \geqslant k}(z)= \begin{cases}0 & \text { if } \nu_{(f, H)}(z)<k, \\
\nu_{(f, H)}(z) & \text { if } \nu_{(f, H)}(z) \geqslant k,\end{cases}
\end{array}
$$

Take a meromorphic mapping $f$ of $\mathbf{C}^{m}$ into $\mathbf{P}^{n}(\mathbf{C})$ which is linearly nondegenerate over $\mathbf{C}$, a positive integer $d$ and $q$ hyperplanes $H_{1}, \ldots, H_{q}$ of $\mathbf{P}^{n}(\mathbf{C})$ in general position with

$$
\operatorname{dim} f^{-1}\left(H_{i} \cap H_{j}\right) \leqslant m-2 \quad(1 \leqslant i<j \leqslant q)
$$

2010 Mathematics Subject Classification: Primary 32H30, 32A22; Secondary 30D35.

Key words and phrases: second main theorem, uniqueness problem, meromorphic mapping, truncated multiplicity. 
and consider the set $\mathcal{F}\left(f,\left\{H_{i}\right\}_{i=1}^{q}, d\right)$ of all linearly nondegenerate over $\mathbf{C}$ meromorphic maps $g: \mathbf{C}^{m} \rightarrow \mathbf{P}^{n}(\mathbf{C})$ satisfying the following two conditions:

(a) $\min \left(\nu_{\left(f, H_{j}\right)}, d\right)=\min \left(\nu_{\left(g, H_{j}\right)}, d\right) \quad(1 \leqslant j \leqslant q)$,

(b) $f(z)=g(z)$ on $\bigcup_{j=1}^{q} f^{-1}\left(H_{j}\right)$.

We see that conditions a) and b) mean the sets of all intersecting points (counted with multiplicity to level $d$ ) of $f$ and $g$ with each hyperplane are the same, and two mappings $f$ and $g$ agree on these sets. If $d=1$, we will say that $f$ and $g$ share $q$ hyperplanes $\left\{H_{j}\right\}_{j=1}^{q}$ regardless of multiplicity.

Denote by $\sharp S$ the cardinality of the set $S$. In 1983, L. Smiley [7] proved the following uniqueness theorem.

Theorem A. If $q=3 n+2$ then $\sharp \mathcal{F}\left(f,\left\{H_{i}\right\}_{i=1}^{q}, 1\right)=1$.

In 1998, H. Fujimoto [2] proved a finiteness theorem for meromorphic mappings as follows.

Theorem B. If $q=3 n+1$ then $\sharp \mathcal{F}\left(f,\left\{H_{i}\right\}_{i=1}^{q}, 2\right) \leqslant 2$.

In 2009, Z. Chen-Q. Yan [1] considered the case of $2 n+3$ hyperplanes and proved the following uniqueness theorem.

Theorem C. If $q=2 n+3$ then $\sharp \mathcal{F}\left(f,\left\{H_{i}\right\}_{i=1}^{q}, 1\right)=1$.

After that, in 2011 S. D. Quang [5] improved the result of Z. Chen-Q. Yan by omitting all zeros with multiplicity more than a certain number in the conditions on sharing hyperplanes of meromorphic mappings. As far as we known, there is still no uniqueness theorem for meromorphic mappings sharing less than $2 n+3$ hyperplanes regardless of multiplicities. In 2011 Q. Yan-Z. Chen [8] also proved a degeneracy theorem as follows.

Theorem D. If $q=2 n+2$ then the map $f^{1} \times f^{2} \times f^{3}$ of $\mathbf{C}^{m}$ into $\mathbf{P}^{N}(\mathbf{C}) \times \mathbf{P}^{N}(\mathbf{C}) \times \mathbf{P}^{N}(\mathbf{C})$ is linearly degenerate for every three maps $f^{1}, f^{2}, f^{3} \in \mathcal{F}\left(f,\left\{H_{i}\right\}_{i=1}^{q}, 2\right)$.

The first finiteness theorem for the case of meromorphic mappings sharing $2 n+2$ hyperplanes regardless of multiplicities are given by S. D. Quang [6] in 2012 as follows.

Theorem E. If $n \geqslant 2$ and $q=2 n+2$ then $\sharp \mathcal{F}\left(f,\left\{H_{i}\right\}_{i=1}^{q}, 1\right) \leqslant 2$.

However we note that there is a gap in the proof of [6, Theorem 1.1]. For detail, the inequality (3.26) in [6, Lemma 3.20] does not holds. Hence the inequality of [ 6 , Lemma 3.20(ii)] may not hold. In order to fix this gap, we need to slightly change the estimate of this inequality by adding $N_{\left(f, H_{j}\right)}^{(1)}(r)$ to its right-hand side. The rest of the proof is still valid for the case where $N \geqslant 3$. In this paper, we will show a correction for [ 6 , Lemma 3.20] (see Lemma 3.9 below). Also this theorem (including the case where $N=2$ ) will be corrected and improved (see Theorem 1.1 below) by another approach.

We would also like to emphasize that in the above results, all intersecting points of the mappings and the hyperplanes are considered. It seems to us that the technique used in the proof of the above results do not work for the case where all such points with multiplicities more than a certain number are not taken to count. Our first purpose in this paper is to improve the above result by omitting all such intersecting points. In order to states the main results, we give the following definition. 
Let $f$ be a linearly nondegenerate meromorphic mapping of $\mathbf{C}^{m}$ into $\mathbf{P}^{n}(\mathbf{C})$ and let $H_{1}, \ldots, H_{q}$ be $q$ hyperplanes of $\mathbf{P}^{n}(\mathbf{C})$ in general position. Let $k_{1}, \ldots, k_{q}$ be $q$ positive integers or $+\infty$. Assume that

$$
\operatorname{dim}\left\{z ; \nu_{\left(f, H_{i}\right), \leqslant k_{i}}(z) \cdot \nu_{\left(f, H_{j}\right), \leqslant k_{j}}(z)>0\right\} \leqslant m-2 \quad(1 \leqslant i<j \leqslant q) .
$$

Let $d$ be an integer. We consider the set $\mathcal{F}\left(f,\left\{H_{i}, k_{i}\right\}_{i=1}^{q}, d\right)$ of all meromorphic maps $g: \mathbf{C}^{m} \rightarrow \mathbf{P}^{n}(\mathbf{C})$ satisfying the conditions:

(a) $\min \left(\nu_{\left(f, H_{i}\right), \leqslant k_{i}}, d\right)=\min \left(\nu_{\left(g, H_{i}\right), \leqslant k_{i}}, d\right) \quad(1 \leqslant j \leqslant q)$,

(b) $f(z)=g(z)$ on $\bigcup_{i=1}^{q}\left\{z ; \nu_{\left(f, H_{i}\right), \leqslant k_{i}}(z)>0\right\}$.

Then we see that $\mathcal{F}\left(f,\left\{H_{i}\right\}_{i=1}^{q}, d\right)=\mathcal{F}\left(f,\left\{H_{i}, \infty\right\}_{i=1}^{q}, d\right)$

Theorem 1.1. Let $f$ be a linearly nondegenerate meromorphic mapping of $\mathbf{C}^{m}$ into $\mathbf{P}^{n}(\mathbf{C})$ $(n \geqslant 2)$. Let $H_{1}, \ldots, H_{2 n+2}$ be $2 n+2$ hyperplanes of $\mathbf{P}^{n}(\mathbf{C})$ in general position and let $k_{1}, \ldots, k_{n+2}$ be positive integers or $+\infty$. Assume that

$$
\begin{gathered}
\operatorname{dim}\left\{z ; \nu_{\left(f, H_{i}\right), \leqslant k_{i}}(z) \cdot \nu_{\left(f, H_{j}\right), \leqslant k_{j}}(z)>0\right\} \leqslant m-2 \quad(1 \leqslant i<j \leqslant 2 n+2), \\
\quad \text { and } \sum_{i=1}^{2 n+2} \frac{1}{k_{i}+1}<\min \left\{\frac{n+1}{3 n^{2}+n}, \frac{5 n-9}{24 n+12}, \frac{n^{2}-1}{10 n^{2}+8 n}\right\} .
\end{gathered}
$$

Then $\sharp \mathcal{F}\left(f,\left\{H_{i}, k_{i}\right\}_{i=1}^{2 n+2}, 1\right) \leqslant 2$.

Then we see that in the case $n \geqslant 2$, Theorems $\mathrm{D}$ and $\mathrm{E}$ are corollaries of Theorem 1.1 when $k_{1}=\cdots=k_{2 n+2}=+\infty$.

The last purpose of this paper is to prove a degeneracy theorem for three mappings sharing $2 n+1$ hyperplanes. Namely, we will proved the following.

Theorem 1.2. Let $f$ be a linearly nondegenerate meromorphic mapping of $\mathbf{C}^{m}$ into $\mathbf{P}^{n}(\mathbf{C})(n \geqslant 5)$. Let $H_{1}, \ldots, H_{2 n+1}$ be $2 n+1$ hyperplanes of $\mathbf{P}^{n}(\mathbf{C})$ in general position and let $k_{1}, \ldots, k_{2 n+1}$ be positive integers or $+\infty$ such that

$$
\operatorname{dim}\left\{z ; \nu_{\left(f, H_{i}\right), \leqslant k_{i}}(z) \cdot \nu_{\left(f, H_{j}\right), \leqslant k_{j}}(z)>0\right\} \leqslant m-2 \quad(1 \leqslant i<j \leqslant 2 n+2) .
$$

If there exists a positive integer $p$ with $p \leqslant n$ and

$$
\sum_{i=1}^{2 n+1} \frac{1}{k_{i}+1}<\frac{n p-3 n-p}{4 n^{2}+3 n p-n} .
$$

then the map $f^{1} \times f^{2} \times f^{3}$ of $\mathbf{C}^{m}$ into $\mathbf{P}^{n}(\mathbf{C}) \times \mathbf{P}^{n}(\mathbf{C}) \times \mathbf{P}^{n}(\mathbf{C})$ is linearly degenerate for every three maps $f^{1}, f^{2}, f^{3} \in \mathcal{F}\left(f,\left\{H_{i}, k_{i}\right\}_{i=1}^{2 n+1}, p\right)$

\section{BASIC NOTIONS IN NEVANLINNA THEORY}

2.1. Counting functions of divisors. We set $\|z\|=\left(\left|z_{1}\right|^{2}+\cdots+\left|z_{m}\right|^{2}\right)^{1 / 2}$ for $z=$ $\left(z_{1}, \ldots, z_{n}\right) \in \mathbf{C}^{m}$ and define

$$
B(r):=\left\{z \in \mathbf{C}^{m}:\|z\|<r\right\}, \quad S(r):=\left\{z \in \mathbf{C}^{m}:\|z\|=r\right\}(0<r<\infty) .
$$

Define

$$
\begin{gathered}
v_{m-1}(z):=\left(d d^{c}\|z\|^{2}\right)^{m-1} \quad \text { and } \\
\sigma_{m}(z):=d^{c} \log \|z\|^{2} \wedge\left(d d^{c} \log \|z\|^{2}\right)^{m-1} \text { on } \quad \mathbf{C}^{m} \backslash\{0\} .
\end{gathered}
$$


We mean by a divisor divisor $\nu$ on a domain $\Omega$ in $\mathbf{C}^{m}$ a formal sum

$$
\nu=\sum_{\lambda \in \Lambda} a_{\lambda} Z_{\lambda}
$$

where $a_{\lambda} \in \mathbf{Z}$ and $\left\{Z_{\lambda}\right\}_{\lambda \in \Lambda}$ is a locally finite family of distinct irreducible hypersurfaces of $\Omega$. Then, we may consider the divisor $\nu$ as a function on $\Omega$ with values in $\mathbf{Z}$ as follows

$$
\nu(z)=\sum_{Z_{\lambda} \ni z} a_{\lambda}
$$

The support of $\nu$ is defined by $\operatorname{Supp} \nu=\bigcup_{a_{\lambda} \neq 0} Z_{\lambda}$.

For a nonzero meromorphic function $\varphi$ on a domain $\Omega$ in $\mathbf{C}^{m}$, we denote by $\nu_{\varphi}^{0}$ (resp. $\nu_{\varphi}^{\infty}$ ) the divisor of zeros (resp. divisor of poles) of $\varphi$, and denote by $\nu_{\varphi}=\nu_{\varphi}^{0}-\nu_{\varphi}^{\infty}$ the divisor generated by $\varphi$.

For a divisor $\nu$ on $\mathbf{C}^{m}$ and for positive integers $k, M$ (or $M=\infty$ ), we define the counting functions of $\nu$ as follows. Set

$$
\begin{gathered}
\nu^{(M)}(z)=\min \{M, \nu(z)\}, \\
\nu_{\leqslant k}^{(M)}(z)= \begin{cases}0 & \text { if } \nu(z)>k, \\
\nu^{(M)}(z) & \text { if } \nu(z) \leqslant k,\end{cases} \\
\nu_{>k}^{(M)}(z)= \begin{cases}\nu^{(M)}(z) & \text { if } \nu(z)>k, \\
0 & \text { if } \nu(z) \leqslant k .\end{cases}
\end{gathered}
$$

We define $n(t)$ by

$$
n(t)= \begin{cases}\int_{|\nu| \cap B(t)} \nu(z) v_{n-1} & \text { if } n \geqslant 2, \\ \sum_{|z| \leqslant t} \nu(z) & \text { if } n=1 .\end{cases}
$$

Similarly, we define $n^{(M)}(t), n_{\leqslant k}^{(M)}(t), n_{>k}^{(M)}(t)$.

Define

$$
N(r, \nu)=\int_{1}^{r} \frac{n(t)}{t^{2 n-1}} d t \quad(1<r<\infty) .
$$

Similarly, we define $N\left(r, \nu^{(M)}\right), N\left(r, \nu_{\leqslant k}^{(M)}\right), N\left(r, \nu_{>k}^{(M)}\right)$ and denote them by $N^{(M)}(r, \nu)$, $N_{\leqslant k}^{(M)}(r, \nu), N_{>k}^{(M)}(r, \nu)$ respectively.

Let $\varphi: \mathbf{C}^{m} \longrightarrow \mathbf{C}$ be a meromorphic function. Define

$$
\begin{gathered}
N_{\varphi}(r)=N\left(r, \nu_{\varphi}^{0}\right), N_{\varphi}^{(M)}(r)=N^{(M)}\left(r, \nu_{\varphi}^{0}\right), \\
N_{\varphi, \leqslant k}^{(M)}(r)=N_{\leqslant k}^{(M)}\left(r, \nu_{\varphi}^{0}\right), N_{\varphi,>k}^{(M)}(r)=N_{>k}^{(M)}\left(r, \nu_{\varphi}^{0}\right) .
\end{gathered}
$$

For brevity we will omit the superscript ${ }^{(M)}$ if $M=\infty$. 
For a set $S \subset \mathbf{C}^{m}$, we define the characteristic function of $S$ by

$$
\chi_{S}(z)= \begin{cases}1 & \text { if } z \in S \\ 0 & \text { if } z \notin S\end{cases}
$$

If the closure $\bar{S}$ of $S$ is an analytic subset of $\mathbf{C}^{m}$ then we denote by $N(r, S)$ the counting function of the reduced divisor whose support is the union of all irreducible components of $\bar{S}$ with codimension one.

2.2. Characteristic and Proximity functions. Let $f: \mathbf{C}^{m} \longrightarrow \mathbf{P}^{n}(\mathbf{C})$ be a meromorphic mapping. For arbitrarily fixed homogeneous coordinates $\left(w_{0}: \cdots: w_{n}\right)$ on $\mathbf{P}^{n}(\mathbf{C})$, we take a reduced representation $f=\left(f_{0}: \cdots: f_{n}\right)$, which means that each $f_{i}$ is a holomorphic function on $\mathbf{C}^{m}$ and $f(z)=\left(f_{0}(z): \cdots: f_{n}(z)\right)$ outside the analytic set $\left\{f_{0}=\cdots=f_{n}=0\right\}$ of codimension $\geqslant 2$. Set $\|f\|=\left(\left|f_{0}\right|^{2}+\cdots+\left|f_{n}\right|^{2}\right)^{1 / 2}$.

The characteristic function of $f$ is defined by

$$
T_{f}(r)=\int_{S(r)} \log \|f\| \sigma_{m}-\int_{S(1)} \log \|f\| \sigma_{m} .
$$

Let $H$ be a hyperplane in $\mathbf{P}^{n}(\mathbf{C})$ given by $H=\left\{a_{0} \omega_{0}+\cdots+a_{n} \omega_{n}\right\}$, where $\left(a_{0}, \ldots, a_{n}\right) \neq$ $(0, \ldots, 0)$. We set $(f, H)=\sum_{i=0}^{n} a_{i} f_{i}$. Then we see that the divisor $\nu_{(f, H)}$ does not depend on the reduced representation of $f$ and presentation of $H$. We define the proximity function of $H$ by

$$
m_{f, H}(r)=\int_{S(r)} \log \frac{\|f\| \cdot\|H\|}{|(f, H)|} \sigma_{m}-\int_{S(1)} \log \frac{\|f\| \cdot\|H\|}{|(f, H)|} \sigma_{m},
$$

where $\|H\|=\left(\sum_{i=0}^{N}\left|a_{i}\right|^{2}\right)^{\frac{1}{2}}$.

Let $\varphi$ be a nonzero meromorphic function on $\mathbf{C}^{m}$, which are occasionally regarded as a meromorphic mapping into $\mathbf{P}^{1}(\mathbf{C})$. The proximity function of $\varphi$ is defined by

$$
m(r, \varphi):=\int_{S(r)} \log \max (|\varphi|, 1) \sigma_{n} .
$$

As usual, by the notation " $\| P$ " we mean the assertion $P$ holds for all $r \in[0, \infty)$ excluding a Borel subset $E$ of the interval $[0, \infty)$ with $\int_{E} d r<\infty$.

2.3. Some lemmas. The following results play essential roles in Nevanlinna theory (see [4]).

Theorem 2.1 (The first main theorem). Let $f: \mathbf{C}^{m} \rightarrow \mathbf{P}^{n}(\mathbf{C})$ be a linearly nondegenerate meromorphic mapping and $H$ be a hyperplane in $\mathbf{P}^{n}(\mathbf{C})$. Then

$$
N_{(f, H)}(r)+m_{f, H}(r)=T_{f}(r)(r>1) .
$$

Theorem 2.2 (The second main theorem). Let $f: \mathbf{C}^{m} \rightarrow \mathbf{P}^{n}(\mathbf{C})$ be a linearly nondegenerate meromorphic mapping and $H_{1}, \ldots, H_{q}$ be hyperplanes in general position in $\mathbf{P}^{n}(\mathbf{C})$. Then

$$
\|(q-n-1) T_{f}(r) \leqslant \sum_{i=1}^{q} N_{\left(f, H_{i}\right)}^{(n)}(r)+o\left(T_{f}(r)\right) .
$$


For meromorphic functions $F, G, H$ on $\mathbf{C}^{m}$ and $\alpha=\left(\alpha_{1}, \ldots, \alpha_{m}\right) \in \mathbf{Z}_{+}^{m}$, we put

$$
\Phi^{\alpha}(F, G, H):=F \cdot G \cdot H \cdot\left|\begin{array}{ccc}
1 & 1 & 1 \\
\frac{1}{F} & \frac{1}{G} & \frac{1}{H} \\
\mathcal{D}^{\alpha}\left(\frac{1}{F}\right) & \mathcal{D}^{\alpha}\left(\frac{1}{G}\right) & \mathcal{D}^{\alpha}\left(\frac{1}{H}\right)
\end{array}\right|
$$

Lemma 2.3 ([2, Proposition 3.4]). If $\Phi^{\alpha}(F, G, H)=0$ and $\Phi^{\alpha}\left(\frac{1}{F}, \frac{1}{G}, \frac{1}{H}\right)=0$ for all $\alpha$ with $|\alpha| \leq 1$, then one of the following assertions holds :

(i) $F=G, G=H$ or $H=F$

(ii) $\frac{F}{G}, \frac{G}{H}$ and $\frac{H}{F}$ are all constant.

Lemma 2.4. Let $f^{1}, f^{2}, f^{3}$ be three maps in $\mathcal{F}\left(f,\left\{H_{i}, k_{i}\right\}_{i=1}^{q}, p\right)$. Assume that $f^{i}$ has a representation $f^{i}=\left(f_{0}^{i}: \cdots: f_{n}^{i}\right), 1 \leqslant i \leqslant 3$. Suppose that there exist $s, t, l \in\{1, \cdots, q\}$ such that

$$
P:=\operatorname{Det}\left(\begin{array}{ccc}
\left(f^{1}, H_{s}\right) & \left(f^{1}, H_{t}\right) & \left(f^{1}, H_{l}\right) \\
\left(f^{2}, H_{s}\right) & \left(f^{2}, H_{t}\right) & \left(f^{2}, H_{l}\right) \\
\left(f^{3}, H_{s}\right) & \left(f^{3}, H_{t}\right) & \left(f^{3}, H_{l}\right)
\end{array}\right) \neq \equiv 0
$$

Then we have

$$
\begin{aligned}
T(r) \geqslant & \sum_{i=s, t, l}\left(N\left(r, \min \left\{\nu_{\left(f^{u}, H_{i}\right), \leqslant k_{i}} ; 1 \leqslant u \leqslant 3\right\}\right)\right. \\
& \left.-N_{\left(f, H_{i}\right), \leq k_{i}}^{(1)}(r)\right)+2 \sum_{i=1}^{q} N_{\left(f, H_{i}\right), \leq k_{i}}^{(1)}(r)+o(T(r)),
\end{aligned}
$$

where $T(r)=\sum_{u=1}^{3} T_{f^{u}}(r)$.

Proof. Denote by $S$ the closure of $\bigcup_{1 \leqslant u \leqslant 3} I\left(f^{u}\right) \cup \bigcup_{1 \leqslant i<j \leqslant 2 n+2}\left\{z ; \nu_{\left(f, H_{i}\right), \leqslant k_{i}}(z) \cdot \nu_{\left(f, H_{j}\right), \leqslant k_{j}}(z)>\right.$ $0\}$. Then $S$ is an analytic subset of codimension two of $\mathbf{C}^{m}$.

For $z \notin S$, we consider the following two cases:

Case 1. $z$ is a zero of $\left(f, H_{i}\right)$ with multiplicity at most $k_{i}$, where $i \in\{s, t, l\}$. For instance, we suppose that $i=s$. We set

$$
m=\min \left\{\nu_{\left(f^{1}, H_{s}\right), \leqslant k_{s}}(z), \nu_{\left(f^{2}, H_{s}\right), \leqslant k_{s}}(z), \nu_{\left(f^{3}, H_{s}\right), \leqslant k_{s}}(z)\right\} .
$$

Then there exist a neighborhood $U$ of $z$ and a holomorphic function $h$ defined on $U$ such that $\operatorname{Zero}(h)=U \cap \operatorname{Zero}\left(f, H_{s}\right)$ and $d h$ has no zero. Then the functions $\varphi_{u}=\frac{\left(f^{u}, H_{s}\right)}{h^{m}}(1 \leqslant$ $u \leqslant 3)$ are holomorphic in a neighborhood of $z$. On the other hand, since $f^{1}=f^{2}=f^{3}$ on $\operatorname{Supp} \nu_{\left(f, H_{s}\right), \leqslant k_{s}}$, we have

$$
P_{u v}:=\left(f^{u}, H_{t}\right)\left(f^{v}, H_{l}\right)-\left(f^{u}, H_{l}\right)\left(f^{v}, H_{t}\right)=0 \text { on } \operatorname{Supp} \nu_{\left(f, H_{s}\right), \leqslant k_{s}}, 1 \leqslant u<v \leqslant 3 .
$$

Therefore, there exist holomorphic functions $\psi_{u v}$ on a neighborhood of $z$ such that $P_{u v}=$ $h \psi_{u v}$. Then we have

$$
P=h^{m+1}\left(\varphi_{1} \psi_{23}-\varphi_{2} \psi_{13}+\varphi_{3} \psi_{12}\right)
$$

on a neighborhood of $z$. This yeilds that

$\nu_{P}(z) \geqslant m+1=\sum_{i=s, t, l}\left(\min \left\{\nu_{\left(f^{u}, H_{i}\right), \leqslant k_{i}}(z) ; 1 \leqslant u \leqslant 3\right\}-\nu_{\left(f, H_{i}\right), \leqslant k_{i}}^{(1)}(z)\right)+2 \sum_{i=1}^{q} \nu_{\left(f, H_{i}\right), \leqslant k_{i}}^{(1)}(z)$. 
Case 2. $z$ is a zero point of $\left(f, H_{i}\right)$ with multiplicity at most $k_{i}$, where $i \notin\{s, t, l\}$. There exist an index $v$ such that $\left(f^{1}, H_{v}\right)(z) \neq 0$. Since $f^{1}(z)=f^{2}(z)=f^{3}(z)$, we have $\left(f^{u}, H_{v}\right)(z) \neq 0(1 \leqslant u \leqslant 3)$ and

$$
\begin{aligned}
P & =\prod_{u=1}^{3}\left(f^{u}, H_{v}\right) \cdot \operatorname{det}\left(\begin{array}{ccc}
\frac{\left(f^{1}, H_{s}\right)}{\left(f^{1}, H_{v}\right)} & \frac{\left(f^{1}, H_{t}\right)}{\left(f^{1}, H_{v}\right)} & \frac{\left(f^{1}, H_{l}\right)}{\left(f^{1}, H_{v}\right)} \\
\frac{\left(f^{2}, H_{1}\right)}{\left(f^{2}, H_{l}\right)} & \frac{\left(f^{2}, H_{t}\right)}{\left(f^{2}, H_{l}\right)} & \frac{\left(f^{2}, H_{s}\right)}{\left(f^{2}, H_{l}\right)} \\
\frac{\left(f^{3}, H_{1}\right)}{\left(f^{3}, H_{l}\right)} & \frac{\left(f^{3}, H_{t}\right)}{\left(f^{3}, H_{l}\right)} & \frac{\left(f^{3}, H_{s}\right)}{\left(f_{3}, H_{l}\right)}
\end{array}\right) \\
& =\prod_{u=1}^{3}\left(f^{u}, H_{l}\right) \cdot \operatorname{det}\left(\begin{array}{ccc}
\frac{\left(f^{1}, H_{1}\right)}{\left(f^{1}, H_{l}\right)} & \frac{\left(f^{1}, H_{t}\right)}{\left(f^{1}, H_{l}\right)} & \frac{\left(f^{1}, H_{s}\right)}{\left(f^{1}, H_{l}\right)} \\
\frac{\left(f^{2}, H_{1}\right)}{\left(f^{2}, H_{l}\right)}-\frac{\left(f^{1}, H_{1}\right)}{\left(f^{1}, H_{l}\right)} & \frac{\left(f^{2}, H_{t}\right)}{\left(f^{2}, H_{l}\right)}-\frac{\left(f^{1}, H_{t}\right)}{\left(f^{1}, H_{l}\right)} & \frac{\left(f^{2}, H_{s}\right)}{\left(f^{2}, H_{l}\right)}-\frac{\left(f^{1}, H_{s}\right)}{\left(f^{1}, H_{l}\right)} \\
\frac{\left(f^{3}, H_{1}\right)}{\left(f^{3}, H_{l}\right)}-\frac{\left(f^{1}, H_{1}\right)}{\left(f^{1}, H_{l}\right)} & \frac{\left(f^{3}, H_{t}\right)}{\left(f^{3}, H_{l}\right)}-\frac{\left(f^{1}, H_{t}\right)}{\left(f^{1}, H_{l}\right)} & \frac{\left(f^{3}, H_{s}\right)}{\left(f^{3}, H_{l}\right)}-\frac{\left(f^{1}, H_{s}\right)}{\left(f^{1}, H_{l}\right)}
\end{array}\right) .
\end{aligned}
$$

vanishes at $z$ with multiplicity at least two. Therefore, we have

$$
\nu_{P}(z) \geqslant 2=\sum_{i=s, t, l}\left(\min \left\{\nu_{\left(f^{u}, H_{i}\right), \leqslant k_{i}}(z) ; 1 \leqslant u \leqslant 3\right\}-\nu_{\left(f, H_{i}\right), \leqslant k_{i}}^{(1)}(z)\right)+2 \sum_{i=1}^{q} \nu_{\left(f, H_{i}\right), \leqslant k_{i}}^{(1)}(z) .
$$

Thus, from the above two cases we have

$$
\nu_{P}(z) \geqslant \sum_{i=s, t, l}\left(\min \left\{\nu_{\left(f^{u}, H_{i}\right), \leqslant k_{i}}(z) ; 1 \leqslant u \leqslant 3\right\}-\nu_{\left(f, H_{i}\right), \leqslant k_{i}}^{(1)}(z)\right)+2 \sum_{i=1}^{q} \nu_{\left(f, H_{i}\right), \leqslant k_{i}}^{(1)}(z),
$$

for all $z$ outside the analytic set $S$. Integrating both sides of the above inequality, we get

$$
\begin{aligned}
N_{P}(r) \geqslant & \sum_{i=s, t, l}\left(N\left(r, \min \left\{\nu_{\left(f^{u}, H_{i}\right), \leqslant k_{i}} ; 1 \leqslant u \leqslant 3\right\}\right)-N_{\left(f, H_{i}\right), \leqslant k_{i}}^{(1)}(r)\right) \\
& +2 \sum_{i=1}^{q} N_{\left(f, H_{i}\right), \leqslant k_{i}}^{(1)}(r)+o(T(r)) .
\end{aligned}
$$

On the other hand, by Jensen's formula and the definition of the characteristic function we have

$$
\begin{aligned}
N_{P}(r) & =\int_{S(r)} \log |P| \sigma_{m}+O(1) \\
& \leqslant \sum_{u=1}^{3} \int_{S(r)} \log \left(\left|\left(f^{u}, H_{1}\right)\right|^{2}+\left|\left(f^{u}, H_{t}\right)\right|^{2}+\left|\left(f^{u}, H_{s}\right)\right|\right)^{\frac{1}{2}} \sigma_{m}+O(1) \\
& \leqslant \sum_{u=1}^{3} \int_{S(r)} \log || f^{u}|| \sigma_{m}+O(1)=T(r)+o(T(r))
\end{aligned}
$$


Thus, we have

$$
\begin{aligned}
T(r) \geqslant & \sum_{i=s, t, l}\left(N\left(r, \min \left\{\nu_{\left(f^{u}, H_{i}\right), \leqslant k_{i}} ; 1 \leqslant u \leqslant 3\right\}\right)-N_{\left(f, H_{i}\right), \leqslant k_{i}}^{(1)}(r)\right) \\
& +2 \sum_{i=1}^{q} N_{\left(f, H_{i}\right), \leqslant k_{i}}^{(1)}(r)+o(T(r)) .
\end{aligned}
$$

The lemma is proved.

\section{Proof of Main Theorems}

Let $f$ be a linearly nondegenerate meromorphic mapping of $\mathbf{C}^{m}$ into $\mathbf{P}^{n}(\mathbf{C})$. Let $H_{1}, \ldots, H_{2 n+2}$ be $2 n+2$ hyperplanes of $\mathbf{P}^{n}(\mathbf{C})$ in general position and let $k_{i} \geqslant n(1 \leqslant$ $i \leqslant 2 n+2)$ be positive integers or $+\infty$ with

$$
\operatorname{dim}\left\{z ; \nu_{\left(f, H_{i}\right), \leqslant k_{i}}(z) \cdot \nu_{\left(f, H_{j}\right), \leqslant k_{j}}(z)>0\right\} \leqslant m-2 \quad(1 \leqslant i<j \leqslant 2 n+2) .
$$

In order to prove Theorem 3.2 , we need the following lemmas.

Lemma 3.1. If $\sum_{i=1}^{2 n+2} \frac{1}{k_{i}+1}<\frac{1}{n}$, then every mapping $g$ in $\mathcal{F}\left(f,\left\{H_{i}, k_{i}\right\}_{i=1}^{2 n+2}, 1\right)$ is linearly nondegenerate and

$$
\| T_{g}(r)=O\left(T_{f}(r)\right) \text { and } \| T_{f}(r)=O\left(T_{g}(r)\right)
$$

Proof. Suppose that there exists a hyperplane $H$ satisfying $g\left(\mathbf{C}^{m}\right) \subset H$. We assume that $f$ and $g$ have reduce representations $f=\left(f_{0}: \cdots: f_{n}\right)$ and $g=\left(g_{0}: \cdots: g_{n}\right)$ respectively. Assume that $H=\left\{\left(\omega_{0}: \cdots: \omega_{n}\right) \mid \sum_{i=0}^{n} a_{i} \omega_{i}=0\right\}$. Since $f$ is linearly nondegenerate, $(f . H) \not \equiv 0$. On the other hand $(f, H)(z)=(g, H)(z)=0$ for all $z \in \bigcup_{i=1}^{2 n+2}\left\{\nu_{\left(f, H_{i}\right), \leqslant k_{i}}\right\}$, hence

$$
N_{(f, H)}(r) \geqslant \sum_{i=1}^{2 n+2} N_{\left(f, H_{i}\right), \leqslant k_{i}}^{(1)}(r) .
$$

It yields that

$$
\begin{aligned}
\| T_{f}(r) & \geqslant N_{(f, H)}(r) \geqslant \sum_{i=1}^{2 n+2} N_{\left(f, H_{i}\right), \leqslant k_{i}}^{(1)}(r)=\sum_{i=1}^{2 n+2}\left(N_{\left(f, H_{i}\right)}^{(1)}(r)-N_{\left(f, H_{i}\right),>k_{i}}^{(1)}(r)\right) \\
& \geqslant \sum_{i=1}^{2 n+2} \frac{1}{n} N_{\left(f, H_{i}\right)}^{(n)}(r)-\sum_{i=1}^{2 n+2} \frac{1}{k_{i}+1} T_{f}(r) \geqslant\left(\frac{n+1}{n}-\sum_{i=1}^{2 n+2} \frac{1}{k_{i}+1}\right) T_{f}(r)+o\left(T_{f}(r)\right) .
\end{aligned}
$$

Letting $r \longrightarrow+\infty$, we get

$$
\sum_{i=1}^{2 n+2} \frac{1}{k_{i}+1} \geqslant \frac{1}{n} .
$$

This is a contradiction. Hence $g\left(\mathbf{C}^{m}\right)$ can not be contained in any hyperplanes of $\mathbf{P}^{n}(\mathbf{C})$. Therefore $g$ is linearly nondegenerate. 
Also by the Second Main Theorem, we have

$$
\begin{aligned}
\|(n+1) T_{g}(r) & \leqslant \sum_{i=1}^{2 n+2} N_{\left(g, H_{i}\right)}^{(n)}(r)+o\left(T_{g}(r)\right) \\
& \leqslant \sum_{i=1}^{2 n+2} n N_{\left(g, H_{i}\right)}^{(1)}(r)+o\left(T_{g}(r)\right) \\
& =\sum_{i=1}^{2 n+2} n\left(N_{\left(g, H_{i}\right), \leqslant k_{i}}^{(1)}(r)+N_{\left(g, H_{i}\right),>k_{i}}^{(1)}(r)\right)+o\left(T_{g}(r)\right) \\
& \leqslant \sum_{i=1}^{2 n+2} n\left(N_{\left(f, H_{i}\right), \leqslant k_{i}}^{(1)}(r)+\frac{1}{k_{i}+1} T_{g}(r)\right)+o\left(T_{g}(r)\right) \\
& \leqslant \sum_{i=1}^{2 n+2} n\left(T_{f}(r)+\frac{1}{k_{i}+1} T_{g}(r)\right)+o\left(T_{f}(r)+T_{g}(r)\right) .
\end{aligned}
$$

Thus

$$
\left(n+1-\sum_{i=1}^{2 n+2} \frac{n}{k_{i}+1}\right) T_{g}(r) \leqslant n(2 n+2) T_{f}(r)+o\left(T_{f}(r)+T_{g}(r)\right) .
$$

We note that

$$
n+1-\sum_{i=1}^{2 n+2} \frac{n}{k_{i}+1}>n>0 .
$$

Hence $\| T_{g}(r)=O\left(T_{f}(r)\right)$. Similarly, we get $\| T_{f}(r)=O\left(T_{g}(r)\right)$.

Lemma 3.2. Assume that $n \geqslant 2$ and

$$
\sum_{i=1}^{2 n+2} \frac{1}{k_{i}+1}<\frac{n+1}{n(3 n+1)}
$$

Then for three maps $f^{1}, f^{2}, f^{3} \in \mathcal{F}\left(f,\left\{H_{i}, k_{i}\right\}_{i=1}^{2 n+2}, 1\right)$ we have $f^{1} \wedge f^{2} \wedge f^{3}=0$.

Proof. By Lemma 3.1, we have that $f^{s}$ is linearly nondegenerate and $\| T_{f^{s}}(r)=O\left(T_{f}(r)\right)$ and $\| T_{f}(r)=O\left(T_{f^{s}}(r)\right)$ for all $s=1,2,3$.

Suppose that $f^{1} \wedge f^{2} \wedge f^{3} \not \equiv 0$. For each $1 \leqslant i \leqslant 2 n+2$, we set

$$
N_{i}(r)=\sum_{u=1}^{3} N_{\left(f^{u}, H_{i}\right), \leqslant k_{i}}^{(n)}(r)-(2 n+1) N_{\left(f, H_{i}\right), \leqslant k_{i}}^{(1)}(r) .
$$

Here, we note that for positive integers $a, b, c$ we have $(\min \{a, b, c\}-1) \geqslant \min \{a, n\}+$ $\min \{a, n\}+\min \{a, n\}-2 n-1$. Then

$$
\min \left\{\nu_{\left(f^{u}, H_{i}\right), \leqslant k_{i}}(z) ; 1 \leqslant u \leqslant 3\right\}-\nu_{\left(f, H_{i}\right), \leqslant k_{i}}^{(1)}(z) \geqslant \sum_{u=1}^{3} \nu_{\left(f^{u}, H_{i}\right), \leqslant k_{i}}^{(n)}(z)-(2 n+1) \nu_{\left(f, H_{i}\right), \leqslant k_{i}}^{(1)}(z)
$$


for all $z \in \operatorname{Supp} \nu_{\left(f, H_{i}\right), \leqslant k_{i}}$. This yeilds that

$$
\begin{aligned}
& N\left(r, \min \left\{\nu_{\left(f^{u}, H_{i}\right), \leqslant k_{i}}(z) ;\right.\right.\leqslant u \leqslant 3\})-N_{\left(f, H_{i}\right), \leqslant k_{i}}^{(1)}(r) \\
& \geqslant \sum_{u=1}^{3} N_{\left(f^{u}, H_{i}\right), \leqslant k_{i}}^{(n)}(r)-(2 n+1) N_{\left(f, H_{i}\right), \leqslant k_{i}}^{(1)}(r)=N_{i}(r) .
\end{aligned}
$$

We denote by $\mathcal{I}$ the set of all permutations of the $(2 n+2)$-tuple $(1, \ldots, 2 n+2)$, that means

$$
\mathcal{I}=\left\{I=\left(i_{1}, \ldots, i_{2 n+2}\right):\left\{i_{1}, \ldots, i_{2 n+2}\right\}=\{1, \ldots, 2 n+2\}\right\}
$$

For each $I=\left(i_{1}, \ldots, i_{2 n+2}\right) \in \mathcal{I}$ we define a subset $E_{I}$ of $[1,+\infty)$ as follows

$$
E_{I}=\left\{r \geqslant 1: N_{i_{1}}(r) \geqslant \cdots \geqslant N_{i_{2 n+2}}(r)\right\}
$$

It is clear that $\bigcup_{I \in \mathcal{I}} E_{I}=[1,+\infty)$. Therefore, there exists an element of $\mathcal{I}$, for instance it is $I_{0}=(1,2, \ldots, 2 n+2)$, satisfying

$$
\int_{E_{i_{0}}} d r=+\infty
$$

Then, we have $N_{1}(r) \geqslant N_{2}(r) \geqslant \cdots \geqslant N_{2 n+2}(r)$ for all $r \in E_{i_{0}}$.

We consider $\mathcal{M}^{3}$ as a vector space over the field $\mathcal{M}$. For each $i=1, \ldots, 2 n+2$, we set

$$
V_{i}=\left(\left(f^{1}, H_{i}\right),\left(f^{2}, H_{i}\right),\left(f^{3}, H_{i}\right)\right) \in \mathcal{M}^{3}
$$

We put

$$
s=\min \left\{i: V_{1} \wedge V_{i} \not \equiv 0\right\}
$$

Since $f^{1} \wedge f^{2} \wedge f^{3} \not \equiv 0$, we have $1<s<n+1$. Also by again $f^{1} \wedge f^{2} \wedge f^{3} \not \equiv 0$, there exists an index $t \in\{s+1, \ldots, n+1\}$ such that $V_{1} \wedge V_{s} \wedge V_{t} \not \equiv 0$. This means that

$$
P:=\operatorname{det}\left(V_{1}, V_{s}, V_{t}\right)=\operatorname{det}\left(\begin{array}{ccc}
\left(f^{1}, H_{1}\right) & \left(f^{1}, H_{s}\right) & \left(f^{1}, H_{t}\right) \\
\left(f^{2}, H_{1}\right) & \left(f^{2}, H_{s}\right) & \left(f^{2}, H_{t}\right) \\
\left(f^{3}, H_{1}\right) & \left(f^{3}, H_{s}\right) & \left(f^{3}, H_{t}\right)
\end{array}\right) \not \equiv 0 .
$$

Set $T(r)=\sum_{u=1}^{3} T_{f^{u}}(r)$. By Lemma 2.4, for $r \in E_{I_{0}}$ we have 


$$
\begin{aligned}
& T(r) \geqslant \sum_{i=1, s, t}\left(N\left(r, \min \left\{\nu_{\left(f^{u}, H_{i}\right), \leqslant k_{i}} ; 1 \leqslant u \leqslant 3\right\}\right)-N_{\left(f, H_{i}\right), \leqslant k_{i}}^{(1)}(r)\right) \\
& +2 \sum_{i=1}^{q} N_{\left(f, H_{i}\right), \leqslant k_{i}}^{(1)}(r)+o(T(r)) \\
& \geqslant N_{1}(r)+N_{s}(r)+2 \sum_{i=1}^{q} N_{\left(f, H_{i}\right)}^{(1)}(r)+o(T(r)) \\
& \geqslant \frac{1}{n+1} \sum_{i=1}^{2 n+2} N_{i}(r)+2 \sum_{i=1}^{2 n+2} N_{\left(f, H_{i}\right), \leqslant k_{i}}^{(1)}(r)+o(T(r)) \text {. } \\
& =\frac{1}{n+1} \sum_{i=1}^{2 n+2}\left(\sum_{u=1}^{3} N_{\left(f^{u}, H_{i}\right), \leqslant k_{i}}^{(n)}(z)-(2 n+1) N_{\left(f, H_{i}\right)}^{(1)}(z)\right)+2 \sum_{i=1}^{2 n+2} N_{\left(f, H_{i}\right), \leqslant k_{i}}^{(1)}(r) \\
& =\frac{1}{n+1} \sum_{i=1}^{2 n+2} \sum_{u=1}^{3} N_{\left(f^{u}, H_{i}\right), \leqslant k_{i}}^{(n)}(z)+\frac{1}{3(n+1)} \sum_{i=1}^{2 n+2} \sum_{u=1}^{3} N_{\left(f^{u}, H_{i}\right), \leqslant k_{i}}^{(1)}(r) \\
& \geqslant\left(1+\frac{1}{3 n}\right) \frac{1}{n+1} \sum_{i=1}^{2 n+2} \sum_{u=1}^{3} N_{\left(f^{u}, H_{i}\right), \leqslant k_{i}}^{(n)}(r) \\
& \geqslant\left(1+\frac{1}{3 n}\right) \frac{1}{n+1} \sum_{i=1}^{2 n+2} \sum_{u=1}^{3}\left(N_{\left(f^{u}, H_{i}\right)}^{(n)}(r)-N_{\left(f^{u}, H_{i}\right),>k_{i}}^{(n)}(r)\right) \\
& \geqslant\left(1+\frac{1}{3 n}\right) \frac{1}{n+1} \sum_{u=1}^{3}\left(n+1-\sum_{i=1}^{2 n+2} \frac{n}{k_{i}+1}\right) T_{f^{u}}(r)+o(T(r)) \\
& =\left(1+\frac{1}{3 n}-\frac{3 n+1}{3(n+1)} \sum_{i=1}^{2 n+2} \frac{1}{k_{i}+1}\right) T(r)+o(T(r)) \text {. }
\end{aligned}
$$

Letting $r \rightarrow+\infty\left(r \in E_{i_{0}}\right)$ we get

$$
1 \geqslant 1+\frac{1}{3 n}-\frac{3 n+1}{3(n+1)} \sum_{i=1}^{2 n+2} \frac{1}{k_{i}+1} .
$$

Thus

$$
\sum_{i=1}^{2 n+2} \frac{1}{k_{i}+1} \geqslant \frac{n+1}{n(3 n+1)} .
$$

This is a contradiction. Hence, $f^{1} \wedge f^{2} \wedge f^{3} \equiv 0$. The lemma is proved.

Now for three mappings $f^{1}, f^{2}, f^{3} \in \mathcal{F}\left(f,\left\{H_{i}, k_{i}\right\}_{i=1}^{2 n+2}, 1\right)$, we define:

$$
\begin{aligned}
F_{k}^{i j} & =\frac{\left(f^{k}, H_{i}\right)}{\left(f^{k}, H_{j}\right)}(0 \leqslant k \leqslant 2,1 \leqslant i, j \leqslant 2 n+2), \\
V_{i} & =\left(\left(f^{1}, H_{i}\right),\left(f^{2}, H_{i}\right),\left(f^{3}, H_{i}\right)\right) \in \mathcal{M}_{m}^{3},
\end{aligned}
$$




$$
\begin{aligned}
T_{i} & =\left\{z ; \nu_{\left(f, H_{i}\right), \leqslant k_{i}}(z)>0\right\}, S_{i}=\bigcup_{u=1}^{3}\left\{z ; \nu_{\left(f_{u}, H_{i}\right),>k_{i}}(z)>0\right\}, \\
R_{i} & =\bigcap_{u=1}^{3}\left\{z ; \nu_{\left(f_{u}, H_{i}\right),>k_{i}}(z)>0\right\}, \\
\nu_{i} & =\left\{z ; k_{i} \geqslant \nu_{\left(f^{u}, H_{i}\right)}(z) \geqslant \nu_{\left(f^{v}, H_{i}\right)}(z)=\nu_{\left(f^{t}, H_{i}\right)}(z) \text { for a permutation }(u, v, t) \text { of }(1,2,3)\right\} .
\end{aligned}
$$

We write $V_{i} \cong V_{j}$ if $V_{i} \wedge V_{j} \equiv 0$, otherwise we write $V_{i} \approx V_{j}$. For $V_{i} \approx V_{j}$, we wirte $V_{i} \sim V_{j}$ if there exist $1 \leqslant u<v \leqslant 3$ such that $F_{u}^{i j}=F_{v}^{i j}$, otherwise we write $V_{i} \not V_{j}$.

Lemma 3.3. With the assumption of Theorem 1.1. Let $h$ and $g$ be two elements of the family $\mathcal{F}\left(f,\left\{H_{i}, k_{i}\right\}_{i=1}^{2 n+2}, 1\right)$. If there exist a constant $\lambda$ and two indices $i, j$ such that $\frac{\left(h, H_{i}\right)}{\left(h, H_{j}\right)}=\lambda \frac{\left(g, H_{i}\right)}{\left(g, H_{j}\right)}$ then $\lambda=1$.

Proof. By Lemma 3.1, we see that $h$ and $g$ are linearly nondegenerate and have the characteristic functions of the same order with the characteristic function of $f$. Setting $H=\frac{\left(h, H_{i}\right)}{\left(h, H_{j}\right)}$ and $G=\frac{\left(g, H_{i}\right)}{\left(g, H_{j}\right)}$ and

$$
S_{t}^{\prime}=\left\{z ; \nu_{\left(h, H_{t}\right),>k_{t}}(z)>0\right\} \cup\left\{z ; \nu_{\left(g, H_{t}\right),>k_{t}}(z)>0\right\} \quad(1 \leqslant t \leqslant 2 n+2) .
$$

Then $H=\lambda G$. Supposing that $\lambda \neq 1$, since $H=G$ on the set $\bigcup_{t \neq i, j} T_{t} \backslash\left(S_{i}^{\prime} \cup S_{j}^{\prime}\right)$, we have $\bigcup_{t \neq i, j} T_{t} \subset S_{i}^{\prime} \cup S_{j}^{\prime}$. Thus

$$
\begin{aligned}
0 & \geq \sum_{t \neq i, j} N_{\left(f, H_{t}\right), \leqslant k_{t}}^{(1)}(r)-\left(N\left(r, S_{i}^{\prime}\right)+N\left(r, S_{j}^{\prime}\right)\right) \\
& \geq \frac{1}{2} \sum_{t \neq i, j}\left(N_{\left(h, H_{t}\right), \leqslant k_{t}}^{(1)}(r)+N_{\left(g, H_{t}\right), \leqslant k_{t}}^{(1)}(r)\right)-\left(N\left(r, S_{i}^{\prime}\right)+N\left(r, S_{j}^{\prime}\right)\right) \\
& \geq \frac{1}{2 n} \sum_{t \neq i, j}\left(N_{\left(h, H_{t}\right)}^{(n)}(r)+N_{\left(g, H_{t}\right)}^{(n)}(r)\right)-\sum_{t=1}^{2 n+2}\left(N_{\left(h, H_{t}\right),>k_{t}}^{(1)}(r)+N_{\left(g, H_{t}\right),>k_{t}}^{(1)}(r)\right) \\
& \geq \frac{n-1}{2 n}\left(T_{h}(r)+T_{g}(r)\right)-\sum_{t=1}^{2 n+2} \frac{1}{k_{t}+1}\left(T_{h}(r)+T_{g}(r)\right)+o\left(T_{f}(r)\right) .
\end{aligned}
$$

Letting $r \longrightarrow+\infty$, we get

$$
\frac{n-1}{2 n} \leqslant \sum_{t=1}^{2 n+2} \frac{1}{k_{t}+1} .
$$

This is a contradiction. Therefore $\lambda=1$. The lemma is proved

Lemma 3.4. Let $f^{1}, f^{2}, f^{3}$ be three elements of $\mathcal{F}\left(f,\left\{H_{i}, k_{i}\right\}_{i=1}^{2 n+2}, 1\right)$, where $k_{i}(1 \leqslant i \leqslant$ $2 n+2)$ are positive integers or $+\infty$. Suppose that $f^{1} \wedge f^{2} \wedge f^{3} \equiv 0$ and $V_{i} \sim V_{j}$ for some distinct indices $i$ and $j$. Then $f^{1}, f^{2}, f^{2}$ are not distinct. 
Proof. Suppose $f^{1}, f^{2}, f^{2}$ are distinct. Since $V_{i} \sim V_{j}$, we may suppose that $F_{1}^{i j}=$ $F_{2}^{i j} \neq F_{3}^{i j}$. Since $f^{1} \wedge f^{2} \wedge f^{3} \equiv 0$ and $f^{1} \neq f^{2}$, there exists a meromorphic function $\alpha$ such that

$$
F_{3}^{t j}=\alpha F_{1}^{t j}+(1-\alpha) F_{2}^{t j}(1 \leqslant t \leqslant 2 n+2) .
$$

This implies that $F_{3}^{i j}=F_{1}^{i j}=F_{2}^{i j}$. This is a contradiction. Hence $f^{1}, f^{2}, f^{3}$ are not distinct. The lemma is proved

Lemma 3.5. With the assumption of Theorem 1.1. Let $f^{1}, f^{2}, f^{3}$ be three maps in $\mathcal{F}\left(f,\left\{H_{i}, k_{i}\right\}_{i=1}^{2 n+2}, 1\right)$. Suppose that $f^{1}, f^{2}, f^{3}$ are distinct and there are two indices $i, j \in$ $\{1,2, \ldots, 2 n+2\}(i \neq j)$ such that $V_{i} \neq V_{j}$ and

$$
\Phi_{i j}^{\alpha}:=\Phi^{\alpha}\left(F_{1}^{i j}, F_{2}^{i j}, F_{3}^{i j}\right) \equiv 0
$$

for every $\alpha=\left(\alpha_{1}, \ldots, \alpha_{m}\right) \in \mathbf{Z}_{+}^{m}$ with $|\alpha|=1$. Then for every $t \in\{1, \ldots, 2 n+2\} \backslash\{i\}$, the following assertion hold:

(i) $\Phi_{i t}^{\alpha} \equiv 0$ for all $|\alpha| \leqslant 1$,

(ii) if $V_{i} \nRightarrow V_{t}$ then $F_{1}^{t i}, F_{2}^{t i}, F_{3}^{t i}$ are distinct and

$$
\begin{aligned}
N_{\left(f, H_{i}\right), \leqslant k_{i}}^{(1)}(r) & \geqslant \sum_{s \neq i, t} N_{\left(f, H_{s}\right), \leqslant k_{s}}^{(1)}(r)-N_{\left(f, H_{t}\right), \leqslant k_{t}}^{(1)}(r)-2\left(N\left(r, S_{i}\right)+N\left(r, S_{t}\right)\right) \\
& \geqslant \sum_{s \neq i, t} N_{\left(f, H_{s}\right), \leqslant k_{s}}^{(1)}(r)-N_{\left(f, H_{t}\right), \leqslant k_{t}}^{(1)}(r)-2 \sum_{u=1}^{3} \sum_{s=i, t} N_{\left(f^{u}, H_{s}\right), \leqslant k_{s}}(r) .
\end{aligned}
$$

Proof. By the supposition $V_{i} ¥ V_{j}$, we may assume that $F_{2}^{j i}-F_{1}^{j i} \neq 0$.

(a) For all $\alpha \in \mathbf{Z}_{+}^{m}$ with $|\alpha|=1$, we have $\Phi_{i j}^{\alpha}=0$, and hence

$$
\begin{aligned}
& \mathcal{D}^{\alpha}\left(\frac{F_{3}^{j i}-F_{1}^{j i}}{F_{2}^{j i}-F_{1}^{j i}}\right)=\frac{1}{\left(F_{2}^{j i}-F_{1}^{j i}\right)^{2}} \cdot\left(\left(F_{2}^{j i}-F_{1}^{j i}\right) \cdot \mathcal{D}^{\alpha}\left(F_{3}^{j i}-F_{1}^{j i}\right)\right. \\
& \left.-\left(F_{3}^{j i}-F_{1}^{j i}\right) \cdot \mathcal{D}^{\alpha}\left(F_{2}^{j i}-F_{1}^{j i}\right)\right) \\
& =\frac{1}{\left(F_{2}^{j i}-F_{1}^{j i}\right)^{2}} \cdot\left|\begin{array}{ccc}
1 & 1 & 1 \\
F_{1}^{j i} & F_{2}^{j i} & F_{3}^{j i} \\
\mathcal{D}^{\alpha}\left(F_{1}^{j i}\right) & \mathcal{D}^{\alpha}\left(F_{2}^{j i}\right) & \mathcal{D}^{\alpha}\left(F_{3}^{j i}\right)
\end{array}\right|=0 .
\end{aligned}
$$

Since the above equality hold for all $|\alpha|=1$, then there exists a constant $c \in \mathbf{C}$ such that

$$
\frac{F_{3}^{j i}-F_{1}^{j i}}{F_{2}^{j i}-F_{1}^{j i}}=c
$$

By Theorem [3.2, we have $f^{1} \wedge f^{2} \wedge f^{3}=0$. Then for each index $t \in\{1, \ldots, 2 n+2\} \backslash\{i, j\}$ we have

$$
\begin{aligned}
0 & =\operatorname{det}\left(\begin{array}{lll}
\left(f_{1}, H_{i}\right) & \left(f_{1}, H_{j}\right) & \left(f_{1}, H_{t}\right) \\
\left(f_{2}, H_{i}\right) & \left(f_{2}, H_{j}\right) & \left(f_{2}, H_{t}\right) \\
\left(f_{3}, H_{i}\right) & \left(f_{3}, H_{j}\right) & \left(f_{3}, H_{t}\right)
\end{array}\right)=\prod_{u=1}^{3}\left(f^{u}, H_{i}\right) \cdot \operatorname{det}\left(\begin{array}{ccc}
1 & F_{1}^{j i} & F_{1}^{t i} \\
1 & F_{2}^{j i} & F_{2}^{t i} \\
1 & F_{3}^{j i} & F_{3}^{t i}
\end{array}\right) \\
& =\prod_{u=1}^{3}\left(f^{u}, H_{i}\right) \cdot \operatorname{det}\left(\begin{array}{cc}
F_{2}^{j i}-F_{1}^{j i} & F_{2}^{t i}-F_{1}^{t i} \\
F_{3}^{j i}-F_{1}^{j i} & F_{3}^{t i}-F_{1}^{t i}
\end{array}\right) .
\end{aligned}
$$


Thus

$$
\left(F_{2}^{j i}-F_{1}^{j i}\right) \cdot\left(F_{3}^{t i}-F_{1}^{t i}\right)=\left(F_{3}^{j i}-F_{1}^{j i}\right) \cdot\left(F_{2}^{t i}-F_{1}^{t i}\right) .
$$

If $F_{2}^{t i}-F_{1}^{t i}=0$ then $F_{3}^{t i}-F_{1}^{t i}=0$, and hence $\Phi_{i t}^{\alpha}=0$ for all $\alpha \in \mathbf{Z}_{+}^{m}$ with $|\alpha|<1$. Otherwise, we have

$$
\frac{F_{3}^{t i}-F_{1}^{t i}}{F_{2}^{t i}-F_{1}^{t i}}=\frac{F_{3}^{j i}-F_{1}^{j i}}{F_{2}^{j i}-F_{1}^{j i}}=c .
$$

This also implies that

$$
\begin{aligned}
\Phi_{i t}^{\alpha} & =F_{1}^{i t} \cdot F_{2}^{i t} \cdot F_{3}^{i t} \cdot\left|\begin{array}{ccc}
1 & 1 & 1 \\
F_{1}^{t i} & F_{2}^{t i} & F_{3}^{t i} \\
\mathcal{D}^{\alpha}\left(F_{1}^{t i}\right) & \mathcal{D}^{\alpha}\left(F_{2}^{t i}\right) & \mathcal{D}^{\alpha}\left(F_{3}^{t i}\right)
\end{array}\right| \\
& =F_{1}^{i t} \cdot F_{2}^{i t} \cdot F_{3}^{i t} \cdot\left|\begin{array}{cc}
F_{2}^{t i}-F_{1}^{t i} & F_{3}^{t i}-F_{1}^{t i} \\
\mathcal{D}^{\alpha}\left(F_{2}^{t i}-F_{1}^{t i}\right) & \mathcal{D}^{\alpha}\left(F_{3}^{t i}-F_{1}^{t i}\right)
\end{array}\right| \\
& =F_{1}^{i t} \cdot F_{2}^{i t} \cdot F_{3}^{i t} \cdot\left|\begin{array}{cc}
F_{2}^{t i}-F_{1}^{t i} & c\left(F_{2}^{t i}-F_{1}^{t i}\right) \\
\mathcal{D}^{\alpha}\left(F_{2}^{t i}-F_{1}^{t i}\right) & c \mathcal{D}^{\alpha}\left(F_{2}^{t i}-F_{1}^{t i}\right)
\end{array}\right|=0 .
\end{aligned}
$$

Then one always has $\Phi_{i t}^{\alpha}=0$ for all $t \in\{1, \ldots, 2 n+2\} \backslash\{i\}$. The first assertion is proved.

(b) We suppose that $V_{i} \nRightarrow V_{t}$. From the above part, we have

$$
c F_{2}^{s i}+(1-c) F_{1}^{s i}=F_{3}^{s i}(s \neq i) .
$$

By the supposition $f^{1}, f^{2}, f^{3}$ are distinct, we have $c \notin\{0,1\}$. This implies that $F_{1}^{t i}, F_{2}^{t i}, F_{3}^{t i}$ are distinct.

We see that the second inequality is clear, then we prove the remain first inequality. We consider the meromorphic mapping $F^{t}$ of $\mathbf{C}^{m}$ into $\mathbf{P}^{1}(\mathbf{C})$ with a reduced representation

$$
F^{t}=\left(F_{1}^{t i} h_{t}: F_{2}^{t i} h_{t}\right)
$$

where $h_{t}$ is a meromorphic function on $\mathbf{C}^{m}$. We see that

$$
\begin{aligned}
T_{F^{t}}(r)= & T\left(r, \frac{F_{1}^{t i}}{F_{2}^{t i}}\right) \leqslant T\left(r, F_{1}^{t i}\right)+T\left(r, \frac{1}{F_{2}^{t i}}\right)+O(1) \\
& \leqslant T\left(r, F_{1}^{t i}\right)+T\left(r, F_{2}^{t i}\right)+O(1) \leqslant T_{f^{1}}(r)+T_{f^{2}}(r)+O(1)=O\left(T_{f}(r)\right) .
\end{aligned}
$$

For a point $z \notin I\left(F^{t}\right) \cup S_{i} \cup S_{t}$ which is a zero of some functions $F_{u}^{t i} h_{t}(1 \leqslant u \leqslant 3)$, then $z$ must be either zero of $\left(f, H_{i}\right)$ with multiplicity at most $k_{i}$ or zero of $\left(f, H_{t}\right)$ with multiplicity at most $k_{t}$, and hence

$$
\sum_{u=1}^{3} \nu_{F_{u}^{t i} h_{t}}^{(1)}(z)=1 \leqslant \nu_{\left(f, H_{i}\right), \leqslant k_{i}}^{(1)}(z)+\nu_{\left(f, H_{t}\right), \leqslant k_{t}}^{(1)}(z) .
$$

This implies that

$$
\sum_{u=1}^{3} \nu_{F_{u}^{t i} h_{t}}^{(1)}(z) \leqslant \nu_{\left(f, H_{i}\right), \leqslant k_{i}}^{(1)}(z)+\nu_{\left(f, H_{t}\right), \leqslant k_{t}}^{(1)}(z)+\chi_{S_{i}}(z)+\chi_{S_{t}}(z)
$$


outside an analytic subset of codimension two. By integrating both sides of this inequality, we get

$$
\sum_{u=1}^{3} N_{F_{u}^{i t} h_{t}}^{(1)}(r) \leqslant N_{\left(f, H_{i}\right) \leqslant k_{i}}^{(1)}(r)+N_{\left(f, H_{t}\right), \leqslant k_{t}}^{(1)}(r)+N\left(r, S_{i}\right)+N\left(r, S_{t}\right)
$$

By the second main theorem, we also have

$$
\| T_{F^{t}}(r) \leqslant \sum_{u=1}^{3} N_{F_{u}^{t i} h_{t}}^{(1)}(r)+o(T(r))
$$

On the other hand, applying the first main theorem to the map $F^{t}$ and the hyperplane $\left\{w_{0}-w_{1}=0\right\}$ in $\mathbf{P}^{1}(\mathbf{C})$, we have

$$
T_{F^{t}}(r) \geqslant N_{\left(F_{1}^{t i}-F_{2}^{t i}\right) h_{t}}(r) \geqslant \sum_{\substack{v=1 \\ v \neq i, t}}^{2 n+2} N_{\left(f, H_{v}\right), \leqslant k_{v}}^{(1)}(r)-N\left(r, S_{i}\right)-N\left(r, S_{t}\right) .
$$

Therefore, from (3.6), (3.7) and (3.8) we have

$$
\| N_{\left(f, H_{i}\right), \leqslant k_{i}}^{(1)}(r) \geqslant \sum_{\substack{v=1 \\ v \neq i, t}}^{2 n+2} N_{\left(f, H_{v}\right), \leqslant k_{v}}^{(1)}(r)-N_{\left(f, H_{t}\right) \leqslant k_{t}}^{(1)}(r)-2\left(N\left(r, S_{i}\right)+N\left(r, S_{t}\right)\right)+o(T(r)) .
$$

The second assertion of the lemma is proved.

Lemma 3.9. With the assumption of Theorem 1.1, let $f^{1}, f^{2}, f^{3}$ be three meromorphic mappings in $\mathcal{F}\left(f,\left\{H_{i}, k_{i}\right\}_{i=1}^{2 n+2}, 1\right)$. Assume that there exist $i, j \in\{1,2, \ldots, 2 n+2\}(i \neq j)$ and $\alpha \in \mathbf{Z}_{+}^{m}$ with $|\alpha|=1$ such that $\Phi_{i j}^{\alpha} \not \equiv 0$. Then we have

$$
\begin{aligned}
T(r) \geqslant & \sum_{u=1}^{3} N_{\left(f^{u}, H_{i}\right), \leqslant k_{i}}^{(n)}(r)+\sum_{k=1}^{3} N_{\left(f^{k}, H_{j}\right), \leqslant k_{j}}^{(n)}(r)+2 \sum_{\substack{t=1 \\
t \neq i, j}}^{2 n+2} N_{\left(f, H_{t}\right), \leqslant k_{t}}^{(1)}(r) \\
& -(2 n+1) N_{\left(f, H_{i}\right), \leqslant k_{i}}^{(1)}(r)-(n+1) N_{\left(f, H_{j}\right), \leqslant k_{j}}^{(1)}(r)+N\left(r, \nu_{j}\right) \\
& -N\left(r, S_{i}\right)-N\left(r, S_{j}\right)-(2 n-2) N\left(r, R_{i}\right)-(n-1) N\left(r, R_{j}\right)+o(T(r)) \\
\geqslant & \sum_{u=1}^{3} N_{\left(f^{u}, H_{i}\right), \leqslant k_{i}}^{(n)}(r)+\sum_{k=1}^{3} N_{\left(f^{k}, H_{j}\right), \leqslant k_{j}}^{(n)}(r)+2 \sum_{\substack{t=1 \\
t \neq i, j}}^{2 n+2} N_{\left(f, H_{t}\right), \leqslant k_{t}}^{(1)}(r) \\
& -(2 n+1) N_{\left(f, H_{i}\right), \leqslant k_{i}}^{(1)}(r)-(n+1) N_{\left(f, H_{j}\right), \leqslant k_{j}}^{(1)}(r)+N\left(r, \nu_{j}\right) \\
& -\sum_{u=1}^{3}\left(\left(1+\frac{n-1}{3}\right) N_{\left(f^{u}, H_{j}\right),>k_{j}}^{(1)}-\left(1+\frac{2 n-2}{3}\right) N_{\left(f^{u}, H_{i}\right),>k_{i}}\right)+o(T(r)) .
\end{aligned}
$$


Proof. The second inequality is clear. We remain prove the first inequality. We have

$$
\begin{aligned}
\Phi^{\alpha} & =F_{1}^{i j} \cdot F_{2}^{i j} \cdot F_{3}^{i j} \cdot\left|\begin{array}{ccc}
1 & 1 & 1 \\
F_{1}^{j i} & F_{2}^{j i} & F_{3}^{j i} \\
\mathcal{D}^{\alpha}\left(F_{1}^{j i}\right) & \mathcal{D}^{\alpha}\left(F_{2}^{j i}\right) & \mathcal{D}^{\alpha}\left(F_{3}^{j i}\right)
\end{array}\right| \\
& =\left|\begin{array}{ccc}
F_{1}^{i j} & F_{2}^{i j} & F_{3}^{i j} \\
1 & 1 & 1 \\
F_{1}^{i j} \mathcal{D}^{\alpha}\left(F_{2}^{j i}\right) & F_{2}^{i j} \mathcal{D}^{\alpha}\left(f^{j i}\right) & F_{3}^{i j} \mathcal{D}^{\alpha}\left(g^{j i}\right)
\end{array}\right|
\end{aligned}
$$

Thus

$$
\begin{aligned}
\Phi_{i j}^{\alpha}= & F_{1}^{i j}\left(\frac{\mathcal{D}^{\alpha}\left(F_{3}^{j i}\right)}{F_{3}^{j i}}-\frac{\mathcal{D}^{\alpha}\left(F_{2}^{j i}\right)}{F_{2}^{j i}}\right)+F_{2}^{i j}\left(\frac{\mathcal{D}^{\alpha}\left(F_{1}^{j i}\right)}{F_{1}^{j i}}-\frac{\mathcal{D}^{\alpha}\left(F_{3}^{j i}\right)}{F_{3}^{j i}}\right) \\
& +F_{3}^{i j}\left(\frac{\mathcal{D}^{\alpha}\left(F_{2}^{j i}\right)}{F_{2}^{j i}}-\frac{\mathcal{D}^{\alpha}\left(F_{1}^{j i}\right)}{F_{1}^{j i}}\right) .
\end{aligned}
$$

By the Logarithmic Derivative Lemma, it follows that

$$
m\left(r, \Phi_{i j}^{\alpha}\right) \leqslant \sum_{u=1}^{3} m\left(r, F_{u}^{i j}\right)+2 \sum_{u=1}^{3} m\left(\frac{\mathcal{D}^{\alpha}\left(F_{u}^{j i}\right)}{F_{v}^{j i}}\right)+O(1) \leqslant \sum_{u=1}^{3} m\left(r, F_{u}^{i j}\right)+o\left(T_{f}(r)\right) .
$$

Therefore, we have

$$
\begin{aligned}
T(r) & \geqslant \sum_{u=1}^{3} T\left(r, F_{u}^{i j}\right)=\sum_{u=1}^{3}\left(m\left(r, F_{u}^{i j}\right)+N_{\frac{1}{F_{u}^{i j}}}(r)\right)=m\left(r, \Phi_{i j}^{\alpha}\right)+\sum_{u=1}^{3} N_{\frac{1}{F_{u}^{i j}}}(r)+o(T(r)) \\
& \geqslant T\left(r, \Phi_{i j}^{\alpha}\right)-N_{\frac{1}{\Phi_{i j}^{\alpha}}}+\sum_{u=1}^{3} N_{\frac{1}{F_{u}^{i j}}}(r)+o(T(r)) \\
& \geqslant N_{\Phi_{i j}^{\alpha}}^{\alpha}(r)-N_{\frac{1}{\Phi_{i j}^{\alpha}}}+\sum_{u=1}^{3} N_{\frac{1}{F_{u u}^{i j}}}(r)+o(T(r)) \\
& =N\left(r, \nu_{\Phi_{i j}^{\alpha}}^{\alpha}\right)+\sum_{u=1}^{3} N_{\frac{1}{F_{u}^{i j}}}(r)+o(T(r)) .
\end{aligned}
$$

Then, in order to prove the lemma, it is sufficient for us to prove

$$
\begin{aligned}
N\left(r, \nu_{\Phi_{i j}^{\alpha}}\right) \geqslant & \sum_{u=1}^{3} N_{\left(f^{u}, H_{i}\right), \leqslant k_{i}}^{(n)}(r)+\sum_{k=1}^{3} N_{\left(f^{k}, H_{j}\right), \leqslant k_{j}}^{(n)}(r)+2 \sum_{\substack{t=1 \\
t \neq i, j}}^{2 n+2} N_{\left(f, H_{t}\right), \leqslant k_{t}}^{(1)}(r) \\
& -(2 n+1) N_{\left(f, H_{i}\right), \leqslant k_{i}}^{(1)}(r)-(n+1) N_{\left(f, H_{j}\right), \leqslant k_{j}}^{(1)}(r)-\sum_{u=1}^{3} N_{\frac{1}{F_{u}^{i j}}}(r)+N\left(r, \nu_{j}\right) \\
& -N\left(r, S_{i}\right)-N\left(r, S_{j}\right)-(2 n-2) N\left(r, R_{i}\right)-(n-1) N\left(r, R_{j}\right)+o(T(r)) .
\end{aligned}
$$

Denote by $S$ the set of all singularities of $f^{-1}\left(H_{t}\right)(1 \leqslant t \leqslant q)$. Then $S$ is an analytic subset of codimension at least two in $\mathbf{C}^{m}$. We set

$$
I=S \cup \bigcup_{s \neq t}\left\{z ; \nu_{\left(f, H_{s}\right), \leqslant k_{s}}(z) \cdot \nu_{\left(f, H_{t}\right), \leqslant k_{t}}(z)>0\right\} .
$$


Then $I$ is also an analytic subset of codimension at least two in $\mathbf{C}^{m}$.

In order to prove the inequality (3.11), it is sufficient for us to show that the following inequality

$$
\begin{aligned}
P: \stackrel{D e f}{=} & \sum_{u=1}^{3} \nu_{\left(f^{u}, H_{i}\right), \leqslant k_{i}}^{(n)}+\sum_{u=1}^{3} \nu_{\left(f^{k}, H_{j}\right) \leqslant k_{j}}^{(n)}+2 \sum_{\substack{t=1 \\
t \neq i, j}}^{2 n+2} \chi_{T_{t}}-(2 n+1) \chi_{T_{i}}-(n+1) \chi_{T_{j}} \\
& -\sum_{u=1}^{3} \nu_{F_{u}^{i j}}^{\infty}+\chi_{\nu_{j}}-\chi_{S_{i}}-\chi_{S_{j}}-2(n-1) \chi_{R_{i}}-(n-1) \chi_{R_{j}} \leqslant \nu_{\Phi_{i j}^{\alpha}} .
\end{aligned}
$$

holds outside the set $I$.

Indeed, for $z \notin I$, we distinguish the following cases:

Case 1: $z \in T_{t} \backslash S_{i} \cup S_{j}(t \neq i, j)$. We see that $P(z)=2$. We write $\Phi_{i j}^{\alpha}$ in the form

$$
\Phi_{i j}^{\alpha}=F_{1}^{i j} \cdot F_{2}^{i j} \cdot F_{3}^{i j} \times\left|\begin{array}{cc}
\left(F_{1}^{j i}-F_{2}^{j i}\right) & \left(F_{1}^{j i}-F_{3}^{j i}\right) \\
\mathcal{D}^{\alpha}\left(F_{1}^{j i}-F_{2}^{j i}\right) & \mathcal{D}^{\alpha}\left(F_{1}^{j i}-F_{3}^{j i}\right)
\end{array}\right|
$$

Then by the assumption that $f^{1}, f^{2}, f^{3}$ are identify on $T_{t}$, we have $F_{1}^{j i}=F_{2}^{j i}=F_{3}^{j i}$ on $T_{t} \backslash S_{i}$. The property of the wronskian implies that $\nu_{\Phi_{i j}^{\alpha}}(z) \geqslant 2=P(z)$.

Case 2: $z \in T_{t} \cap\left(S_{i} \cup S_{j}\right)(t \neq i, j)$. We see that $P(z) \leqslant-\sum_{u=1}^{3} \nu_{F_{u}^{i j}}^{\infty}(z)-1$.

From (3.10) we see that

$$
\nu_{\Phi_{i j}^{\alpha}}(z) \geqslant \min \left\{\nu_{F_{1}^{i j}}(z)-1, \nu_{F_{2}^{i j}}(z)-1, \nu_{F_{3}^{i j}}(z)-1\right\} \geqslant P(z) .
$$

Case 3: $z \in T_{i} \backslash S_{j}$. We have

$$
P(z)=\sum_{u=1}^{3} \nu_{\left(f^{u}, H_{i}\right), \leqslant k_{i}}^{(n)}(z)-(2 n+1) \leqslant \min _{1 \leqslant u \leqslant 3}\left\{\nu_{\left(f^{u}, H_{i}\right), \leqslant k_{i}}^{(n)}(z)\right\}-1 .
$$

We may assume that $\nu_{\left(f^{1}, H_{i}\right)}(z) \leqslant \nu_{\left(f^{2}, H_{i}\right)}(z) \leqslant \nu_{\left(f^{3}, H_{i}\right)}(z)$. We write

$$
\Phi_{i j}^{\alpha}=F_{1}^{i j}\left[F_{2}^{i j}\left(F_{1}^{j i}-F_{2}^{j i}\right) F_{3}^{i j} \mathcal{D}^{\alpha}\left(F_{1}^{j i}-F_{3}^{j i}\right)-F_{3}^{i j}\left(F_{1}^{j i}-F_{2}^{j i}\right) F_{2}^{i j} \mathcal{D}^{\alpha}\left(F_{1}^{j i}-F_{2}^{j i}\right)\right]
$$

It is easy to see that $F_{2}^{i j}\left(F_{1}^{j i}-F_{2}^{j i}\right)$ and $F_{3}^{i j}\left(F_{1}^{j i}-F_{3}^{j i}\right)$ are holomorphic on a neighborhood of $z$ and

$$
\nu_{F_{3}^{i j} \mathcal{D}^{\alpha}\left(F_{1}^{j i}-F_{3}^{j i}\right)}^{\infty}(z) \leqslant 1
$$

and

$$
\nu_{F_{2}^{i j} \mathcal{D}^{\alpha}\left(F_{1}^{j i}-F_{2}^{j i}\right)}^{\infty}(z) \leqslant 1 .
$$

Therefore, it implies that

$$
\nu_{\Phi_{i j}^{\alpha}}(z) \geqslant \nu_{\left(f^{1}, H_{i}\right), \leqslant k_{i}}^{(n)}(z)-1 \geqslant P(z) .
$$


Case 4: $z \in T_{i} \cap S_{j}$. The assumption that $f^{1}, f^{2}, f^{3}$ are identity on $T_{i}$ yields that $z \in R_{j}$. We have

$$
P(z) \leqslant \sum_{u=1}^{3} \nu_{\left(f^{u}, H_{i}\right), \leqslant k_{i}}^{(n)}(z)-\sum_{u=1}^{3} \nu_{F_{u}^{i j}}^{\infty}(z)-(2 n+1)-n \leqslant-\sum_{u=1}^{3} \nu_{F_{u}^{i j}}^{\infty}(z)-1 .
$$

We have

$$
\nu_{\Phi_{i j}^{\alpha}}(z) \geqslant \min \left\{\nu_{F_{1}^{i j}}(z)-1, \nu_{F_{2}^{i j}}(z)-1, \nu_{F_{3}^{i j}}(z)-1\right\} \geqslant-\sum_{u=1}^{3} \nu_{F_{u}^{i j}}^{\infty}(z)-1 \geqslant P(z) .
$$

Case 5: $z \in T_{j}$. We may assume that

$$
\nu_{F_{1}^{j i}}(z)=d_{1} \geqslant \nu_{F_{2}^{j i}}(z)=d_{2} \geqslant \nu_{F_{3}^{j i}}(z)=d_{3} .
$$

Choose a holomorphic function $h$ on $\mathbf{C}^{m}$ with multiplicity 1 at $z$ such that $F_{u}^{j i}=$ $h^{d_{u}} \varphi_{u}(1 \leqslant u \leqslant 3)$, where $\varphi_{u}$ are meromorphic on $\mathbf{C}^{m}$ and holomorphic on a neighborhood of $z$. Then

$$
\begin{aligned}
\Phi_{i j}^{\alpha} & =F_{1}^{i j} \cdot F_{2}^{i j} \cdot F_{3}^{i j} \cdot\left|\begin{array}{cc}
F_{2}^{j i}-F_{1}^{j i} & F_{3}^{j i}-F_{1}^{j i} \\
\mathcal{D}^{\alpha}\left(F_{2}^{j i}-F_{1}^{j i}\right) & \mathcal{D}^{\alpha}\left(F_{3}^{j i}-F_{1}^{j i}\right)
\end{array}\right| \\
& =F_{1}^{i j} \cdot F_{2}^{i j} \cdot F_{3}^{i j} \cdot h^{d_{2}+d_{3}} \cdot\left|\begin{array}{cc}
\varphi_{2}-h^{d_{1}-d_{2}} \varphi_{1} & \varphi_{3}-h^{d_{1}-d_{3}} \varphi_{1} \\
\frac{\mathcal{D}^{\alpha}\left(h^{d_{2}-d_{3}} \varphi_{2}-h^{d_{1}-d_{3}} \varphi_{1}\right)}{h^{d_{2}-d_{3}}} & \mathcal{D}^{\alpha}\left(\varphi_{3}-h^{d_{1}-d_{3}} \varphi_{1}\right)
\end{array}\right| .
\end{aligned}
$$

This yields that

$$
\nu_{\Phi_{i j}^{\alpha}}(z) \geqslant \sum_{u=1}^{3} \nu_{F_{u}^{i j}}(z)+d_{2}+d_{3}-\max \left\{0, \min \left\{1, d_{2}-d_{3}\right\}\right\} .
$$

If $z \notin S_{i}$ then

$$
P(z)=-\sum_{u=1}^{3} \nu_{F_{u}^{i j}}^{\infty}(z)+\sum_{u=1}^{3} \min \left\{n, d_{u}\right\}-(n+1)+\chi_{\nu_{j}}
$$

and

$$
\begin{aligned}
\nu_{\Phi_{i j}^{\alpha}}(z) & \geqslant-\sum_{u=1}^{3} \nu_{F_{u}^{i j}}^{\infty}(z)+\sum_{u=1}^{3} \nu_{F_{u}^{i j}}^{0}(z)+d_{2}+d_{3}-1+\chi_{\nu_{j}} \\
& \geqslant-\sum_{u=1}^{3} \nu_{F_{u}^{i j}}^{\infty}(z)+d_{2}+d_{3}-1+\chi_{\nu_{j}} \geqslant P(z) .
\end{aligned}
$$

Otherwise, if $z \in S_{i}$ then $z \in R_{i}$, and hence

$$
P(z) \leqslant \sum_{u=1}^{3} \nu_{\left(f^{u}, H_{j}\right), \leqslant k_{j}}^{(n)}-\sum_{u=1}^{3} \nu_{F_{u}^{i j}}^{\infty}(z)-3 n-1+\chi_{\nu_{j}} \leqslant-\sum_{u=1}^{3} \nu_{F_{u}^{i j}}^{\infty}(z)-3 n,
$$


and $\begin{aligned} \nu_{\Phi_{i j}^{\alpha}}(z) & \geqslant-\sum_{u=1}^{3} \nu_{F_{u}^{i j}}^{\infty}(z)+\sum_{u=1}^{3} \nu_{F_{u}^{i j}}^{0}(z)+d_{2}+d_{3}-1 \\ & \geqslant-\sum_{u=1}^{3} \nu_{F_{u}^{i j}}^{\infty}(z)+\max \left\{0,-d_{1}\right\}+\max \left\{d_{2}, 0\right\}+\max \left\{d_{3}, 0\right\}-1 \geqslant P(z) .\end{aligned}$

Case 6: $z \in\left(S_{i} \cup S_{j}\right) \backslash\left(\bigcup_{t=1}^{2 n+2} T_{t}\right)$. Similarly as Case 5 , we have

$$
\begin{aligned}
\nu_{\Phi_{i j}^{\alpha}}(z) & \geqslant-\sum_{u=1}^{3} \nu_{F_{u}^{i j}}^{\infty}(z)+\max \left\{0,-d_{1}\right\}+\max \left\{d_{2}, 0\right\}+\max \left\{d_{3}, 0\right\}-1 \\
& \geq-\sum_{u=1}^{3} \nu_{F_{u}^{i j}}^{\infty}(z)-1 \geqslant-\sum_{u=1}^{3} \nu_{F_{u}^{i j}}^{\infty}(z)-\chi_{S_{i}}-\chi_{S_{j}} \geqslant P(z) .
\end{aligned}
$$

From the above six cases, we see that the inequality (3.12) holds. Hence the lemma is proved

Proof of theorem 1.1. Suppose that there exits three distinct meromorphic mappings $f^{1}, f^{2}, f^{3}$ in $\mathcal{F}\left(f,\left\{H_{i}, k_{i}\right\}_{i=1}^{2 n+2}, 1\right)$. By Lemma 3.2, we have $f^{1} \wedge f^{2} \wedge f^{3} \equiv 0$. Without loss of generality, we may assume that

$$
\underbrace{V_{1} \cong \cdots \cong V_{l_{1}}}_{\text {group } 1} \nRightarrow \underbrace{V_{l_{1}+1} \cong \cdots \cong V_{l_{2}}}_{\text {group } 2} \not \equiv \underbrace{V_{l_{2}+1} \cong \cdots \cong V_{l_{3}}}_{\text {group } 3} \nRightarrow \cdots \neq \underbrace{V_{l_{s}+1} \cong \cdots \cong V_{l_{s+1}}}_{\text {group } s} \text {, }
$$

where $l_{s}=2 n+2$.

Denote by $P$ the set of all $i \in\{1, \ldots, 2 n+2\}$ satisfying there exist $j \in\{1, \ldots, 2 n+2\} \backslash\{i\}$ such that $V_{i} \neq V_{j}$ and $\Phi_{i j}^{\alpha} \equiv 0$ for all $\alpha \in \mathbf{Z}_{+}^{m}$ with $|\alpha| \leqslant 1$. We consider the following three cases.

Case 1: $\sharp P \geqslant 2$. Then $P$ contains two elements $i, j$. Then we have $\Phi_{i j}^{\alpha}=\Phi_{j i}^{\alpha}=0$ for all $\alpha \in \mathbf{Z}_{+}^{m}$ with $|\alpha| \leqslant 1$. By Lemma 2.3 , there exist two functions, for instance they are $F_{1}^{i j}$ and $F_{i j}^{2}$, and a constant $\lambda$ such that $F_{1}^{i j}=\lambda F_{2}^{i j}$. This yields that $F_{1}^{i j}=F_{2}^{i j}$ (by Lemma 3.3). Then by Lemma 3.5 (ii), we easily see that $V_{i} \cong V_{j}$, i.e., $V_{i}$ and $V_{j}$ belong to the same group in the above partition.

Without loss of generality, we may assum that $i=1$ and $j=2$. Since $f^{1}, f^{2}, f^{3}$ are supposed to be distinct, the number of each group in the above partition is less than $n+1$. Hence we have $V_{1} \cong V_{2} \neq V_{t}$ for all $t \in\{n+1, \ldots, 2 n+2\}$. Then by Lemma 3.5 (ii), we have

$$
\begin{aligned}
& N_{\left(f, H_{i}\right), \leqslant k_{1}}^{(1)}(r)+N_{\left(f, H_{t}\right), \leqslant k_{t}}^{(1)}(r) \geqslant \sum_{s \neq 1, t} N_{\left(f, H_{s}\right), \leqslant k_{s}}^{(1)}(r)-2 \sum_{u=1}^{3} \sum_{s=1, t} N_{\left(f^{u}, H_{s}\right),>k_{s}}^{(1)}(r), \\
& \text { and } N_{\left(f, H_{2}\right), \leqslant k_{2}}^{(1)}(r)+N_{\left(f, H_{t}\right), \leqslant k_{t}}^{(1)}(r) \geqslant \sum_{s \neq 2, t} N_{\left(f, H_{s}\right), \leqslant k_{s}}^{(1)}(r)-2 \sum_{u=1}^{3} \sum_{s=2, t} N_{\left(f^{u}, H_{s}\right),>k_{s}}^{(1)}(r) .
\end{aligned}
$$


Summing-up both sides of the above two inequalities, we get

$$
\begin{aligned}
2 N_{\left(f, H_{t}\right), \leqslant k_{t}}^{(1)}(r) \geq & 2 \sum_{s \neq 1,2, t} N_{\left(f, H_{s}\right), \leqslant k_{s}}^{(1)}(r)-2 \sum_{u=1}^{3}\left(N_{\left(f^{u}, H_{1}\right),>k_{1}}^{(1)}(r)+N_{\left(f^{u}, H_{2}\right),>k_{2}}^{(1)}(r)\right. \\
& \left.+2 N_{\left(f^{u}, H_{t}\right),>k_{t}}^{(1)}(r)\right) .
\end{aligned}
$$

After summing-up both sides of the above inequalities over all $t \in\{n+1,2 n+2\}$, we easily obtain

$$
\begin{aligned}
\| \sum_{u=1}^{3}((n+2) & \left.\left(N_{\left(f^{u}, H_{1}\right),>k_{1}}^{(1)}(r)+N_{\left(f^{u}, H_{2}\right),>k_{2}}^{(1)}(r)\right)+2 \sum_{t=n+1}^{2 n+2} N_{\left(f^{u}, H_{t}\right),>k_{t}}^{(1)}(r)\right) \\
& \geqslant(n+2) \sum_{t=3}^{n} N_{\left(f, H_{t}\right), \leqslant k_{t}}^{(1)}(r)+n \sum_{t=n+1}^{2 n+2} N_{\left(f, H_{t}\right), \leqslant k_{t}}^{(1)}(r) \\
& \geqslant n \sum_{t=3}^{2 n+2} N_{\left(f, H_{t}\right), \leqslant k_{t}}^{(1)}(r) \geqslant \frac{n}{3} \sum_{u=1}^{3} \sum_{t=3}^{2 n+2} N_{\left(f_{u}, H_{t}\right), \leqslant k_{t}}^{(1)}(r) \\
& \geqslant \frac{n}{3} \sum_{u=1}^{3} \sum_{t=3}^{2 n+2} N_{\left(f_{u}, H_{t}\right)}^{(1)}(r)-\frac{n}{3} \sum_{u=1}^{3} \sum_{t=3}^{2 n+2} N_{\left(f^{u}, H_{t}\right),>k_{t}}^{(1)}(r) \\
& \geqslant \frac{1}{3} \sum_{u=1}^{3} \sum_{t=3}^{2 n+2} N_{\left(f_{u}, H_{t}\right)}^{(n)}(r)-\frac{n}{3} \sum_{u=1}^{3} \sum_{t=3}^{2 n+2} N_{\left(f^{u}, H_{t}\right),>k_{t}}^{(1)}(r) \\
& \geqslant \frac{n-1}{3} T(r)-\frac{n}{3} \sum_{u=1}^{3} \sum_{t=3}^{2 n+2} N_{\left(f^{u}, H_{t}\right),>k_{t}}^{(1)}(r)+o(T(r)) .
\end{aligned}
$$

Therefore, we have

$$
\begin{aligned}
\frac{n-1}{3} T(r) & \leqslant(n+2) \sum_{u=1}^{3} \sum_{t=1}^{2 n+2} N_{\left(f^{u}, H_{t}\right),>k_{t}}^{(1)}(r) \leqslant(n+2) \sum_{u=1}^{3} \sum_{t=1}^{2 n+2} \frac{1}{k_{t}+1} N_{\left(f^{u}, H_{t}\right),>k_{t}}(r) \\
& \leqslant(n+2) \sum_{t=1}^{2 n+2} \frac{1}{k_{t}+1} T(r) .
\end{aligned}
$$

Letting $r \longrightarrow+\infty$, we get

$$
\frac{n-1}{3(n+2)} \leqslant \sum_{t=1}^{2 n+2} \frac{1}{k_{t}+1}
$$

This is a contradiction.

Case 2: $\sharp P=1$. We assume that $P=\{1\}$. We easily see that $V_{1} ¥ V_{i}$ for all $i=2, \ldots, 2 n+2$ (otherwise $i \in P$, this contradict to $\sharp P=1$ ). Then by Lemma 3.5 (ii), we have

$$
N_{\left(f, H_{1}\right), \leqslant k_{1}}^{(1)}(r) \geqslant \sum_{s \neq 1, i} N_{\left(f, H_{s}\right), \leqslant k_{s}}^{(1)}(r)-N_{\left(f, H_{i}\right), \leqslant k_{i}}^{(1)}(r)-2 \sum_{u=1}^{3} \sum_{s=1, i} N_{\left(f^{u}, H_{s}\right),>k_{s}}^{(1)}(r)+o(T(r)) .
$$


Summing-up both sides of the above inequality over all $i=2, \ldots, 2 n+2$, we get

$$
\begin{aligned}
(2 n+1) N_{\left(f, H_{1}\right), \leqslant k_{1}}^{(1)}(r) \geq & (2 n-1) \sum_{i=2}^{2 n+2} N_{\left(f, H_{i}\right), \leqslant k_{i}}^{(1)}(r)-2 \sum_{u=1}^{3} \sum_{i=2}^{2 n+2} N_{\left(f^{u}, H_{i}\right),>k_{s}}^{(1)}(r) \\
& -2(2 n+1) \sum_{u=1}^{3} N_{\left(f^{u}, H_{1}\right),>k_{1}}^{(1)}(r)+o(T(r))
\end{aligned}
$$

We also see that $i \notin P$ for all $2 \leqslant i \leqslant 2 n+2$. We set

$$
\sigma(i)= \begin{cases}i+n & \text { if } i \leqslant n+2 \\ i-n & \text { if } n+2<i \leqslant 2 n+2\end{cases}
$$

Then we easily see that $i$ and $\sigma(i)$ belong to two distinct groups, i.e, $V_{i} ¥ V_{\sigma(i)}$, for all $i \in\{2, \ldots, 2 n+2\}$, and hence $\Phi_{i \sigma(i)}^{\alpha} \not \equiv 0$ for all $\alpha \in \mathbf{Z}_{+}^{m}$ with $|\alpha| \leqslant 1$. By Lemma 3.6 we have

$$
\begin{aligned}
T(r) \geq & \sum_{u=1}^{3} \sum_{t=i, \sigma(i)} N_{\left(f^{u}, H_{t}\right), \leqslant k_{t}}^{(n)}(r)-(2 n+1) N_{\left(f, H_{i}\right), \leqslant k_{i}}^{(1)}(r)-(n+1) N_{\left(f, H_{\sigma(i)}\right) \leqslant k_{\sigma(i)}}^{(1)}(r) \\
& +2 \sum_{\substack{t=1 \\
t \neq i, \sigma(i)}}^{2 n+2} N_{\left(f, H_{t}\right), \leqslant k_{t}}^{(1)}(r)-\sum_{u=1}^{3}\left(\frac{2 n+1}{3} N_{\left(f^{u}, H_{i}\right),>k_{i}}^{(1)}(r)+\frac{n+2}{3} N_{\left(f^{u}, H_{\sigma(i)}\right),>k_{\sigma(i)}}^{(1)}\right) \\
& +o(T(r)) .
\end{aligned}
$$

Summing-up both sides of the above inequalities over all $i \in\{2, \ldots, 2 n+2\}$, we get

$$
\begin{aligned}
(2 n+1) T(r) \geqslant & 2 \sum_{i=2}^{2 n+2} \sum_{u=1}^{3} N_{\left(f^{u}, H_{i}\right), \leqslant k_{i}}^{(n)}(r)+(n-4) \sum_{i=2}^{2 n+2} N_{\left(f, H_{i}\right), \leqslant k_{i}}^{(1)}(r) \\
& +2(2 n+1) N_{\left(f, H_{1}\right), \leqslant k_{1}}^{(1)}(r)-(n+1) \sum_{u=1}^{3} \sum_{i=2}^{2 n+2} N_{\left(f^{u}, H_{i}\right),>k_{i}}^{(1)}+o(T(r)) \\
\geqslant & 2 \sum_{i=2}^{2 n+2} \sum_{u=1}^{3} N_{\left(f^{u}, H_{i}\right), \leqslant k_{i}}^{(n)}(r)+\frac{5 n-6}{3} \sum_{u=1}^{3} \sum_{i=2}^{2 n+2} N_{\left(f^{u}, H_{i}\right), \leqslant k_{i}}^{(1)}(r) \\
& -(8 n+4) \sum_{u=1}^{3} N_{\left(f^{u}, H_{1}\right),>k_{1}}^{(1)}(r)-(n+5) \sum_{u=1}^{3} \sum_{i=2}^{2 n+2} N_{\left(f^{u}, H_{i}\right),>k_{i}}^{(1)}+o(T(r))+o(T(r)) \\
\geqslant & \frac{11 n-6}{3 n} \sum_{u=1}^{3} \sum_{i=1}^{2 n+2} N_{\left(f^{u}, H_{i}\right), \leqslant k_{i}}^{(n)}(r)
\end{aligned}
$$




$$
\begin{aligned}
& -\frac{4 n+2}{3} \sum_{u=1}^{3} N_{\left(f^{u}, H_{1}\right),>k_{1}}^{(1)}(r)-(n+1) \sum_{u=1}^{3} \sum_{i=2}^{2 n+2} N_{\left(f^{u}, H_{i}\right),>k_{i}}^{(1)}+o(T(r))+o(T(r)) \\
& \geqslant \frac{11 n-6}{3 n} \sum_{u=1}^{3} \sum_{i=2}^{2 n+2} N_{\left(f^{u}, H_{i}\right)}^{(n)}(r) \\
& -(8 n+4) \sum_{u=1}^{3} N_{\left(f^{u}, H_{1}\right),>k_{1}}^{(1)}(r)-\frac{14 n+3}{3} \sum_{u=1}^{3} \sum_{i=2}^{2 n+2} N_{\left(f^{u}, H_{i}\right),>k_{i}}^{(1)}+o(T(r))+o(T(r)) \\
& \geqslant \frac{11 n-6}{3} T(r)-(8 n+4) \sum_{i=1}^{2 n+2} \frac{1}{k_{i}+1} T(r)+o(T(r)) .
\end{aligned}
$$

Letting $r \longrightarrow+\infty$, we get

$$
\frac{5 n-9}{24 n+12} \leqslant \sum_{i=1}^{2 n+2} \frac{1}{k_{i}+1}
$$

This is a contradiction.

Case 3: $P=\emptyset$. Then for all $i \neq j$, by Lemma 3.6 we have

$$
\begin{aligned}
T(r) \geqslant & \sum_{u=1}^{3} N_{\left(f^{u}, H_{i}\right), \leqslant k_{i}}^{(n)}(r)+\sum_{k=1}^{3} N_{\left(f^{k}, H_{j}\right), \leqslant k_{j}}^{(n)}(r)+2 \sum_{\substack{t=1 \\
t \neq i, j}}^{2 n+2} N_{\left(f, H_{t}\right), \leqslant k_{t}}^{(1)}(r) \\
& -(2 n+1) N_{\left(f, H_{i}\right), \leqslant k_{i}}^{(1)}(r)-(n+1) N_{\left(f, H_{j}\right), \leqslant k_{j}}^{(1)}(r)+N\left(r, \nu_{j}\right) \\
& -\sum_{u=1}^{3}\left(\left(1+\frac{n-1}{3}\right) N_{\left(f^{u}, H_{j}\right),>k_{j}}^{(1)}(r)+\left(1+\frac{2 n-2}{3}\right) N_{\left(f^{u}, H_{i}\right),>k_{i}}^{(1)}(r)\right)+o(T(r)) .
\end{aligned}
$$

Summing-up both sides of the above inequalities over all pairs $(i, j)$ we get

$$
\begin{aligned}
(2 n+2) T(r) \geqslant & 2 \sum_{u=1}^{3} \sum_{t=1}^{2 n+2} N_{\left(f^{u}, H_{t}\right), \leqslant k_{t}}^{(n)}(r)+(n-2) \sum_{t=1}^{2 n+2} N_{\left(f, H_{t}\right), \leqslant k_{t}}^{(1)}(r)+\sum_{t=1}^{2 n+2} N\left(r, \nu_{t}\right) \\
& -(n+1) \sum_{u=1}^{3} \sum_{t=1}^{2 n+2} N_{\left(f^{u}, H_{i}\right),>k_{i}}+o(T(r)) .
\end{aligned}
$$

On the other hand, by Lemma [3.4, we see that $V_{j} \not V_{l}$ for all $j \neq l$. Hence, we have

$$
P_{s t}^{j l}: \stackrel{\text { Def }}{=}\left(f^{s}, H_{j}\right)\left(f^{t}, H_{l}\right)-\left(f^{t}, H_{l}\right)\left(f^{s}, H_{j}\right) \not \equiv 0(s \neq t, j \neq l) .
$$

Claim 3.15. With $i \neq j \neq l \neq i$, for every $z \in T_{i}$ we have

$$
\sum_{1 \leqslant s<t \leqslant 3} \nu_{P_{s t}^{j l}}(z) \geqslant 4 \chi_{T_{i}}(z)-\chi_{\nu_{i}}(z) .
$$


Indeed, for $z \in T_{i} \backslash \nu_{i}$, we may assume that $\nu_{\left(f^{1}, H_{i}\right)}(z)<\nu_{\left(f^{2}, H_{i}\right)}(z) \leqslant \nu_{\left(f^{3}, H_{i}\right)}(z)$. Since $f^{1} \wedge f^{2} \wedge f^{3} \equiv 0$, we have $\operatorname{det}\left(V_{i}, V_{j}, V_{l}\right) \equiv 0$, and hence

$$
\left(f^{1}, H_{i}\right) P_{23}^{j l}=\left(f^{2}, H_{i}\right) P_{13}^{j l}-\left(f^{3}, H_{i}\right) P_{12}^{j l} .
$$

This yields that

$$
\nu_{P_{23}^{j l}}(z) \geqslant 2
$$

and hence $\sum_{1 \leqslant s<t \leqslant 3} \nu_{P_{s t}^{j l}}(z) \geqslant 4=4 \chi_{T_{i}}(z)-\chi_{\nu_{i}}(z)$.

Now, for $z \in \nu_{i}$, we have $\sum_{1 \leqslant s<t \leqslant 3} \nu_{P_{s t}^{j l}}(z) \geqslant 3=4 \chi_{T_{i}}(z)-\chi_{\nu_{i}}(z)$. Hence, the claim is proved.

On the other hand, with $i=j$ or $i=l$, for every $z \in\left\{\nu_{\left(f, H_{i}\right), \leqslant k_{i}}(z)>0\right\}$ we see that

$$
\begin{aligned}
\nu_{P_{s t}^{j l}}^{j l}(z) & \geq \min \left\{\nu_{\left(f^{s}, H_{i}\right), \leqslant k_{i}}(z), \nu_{\left(f^{t}, H_{i}\right), \leqslant k_{i}}(z)\right\} \\
& \geq \nu_{\left(f^{s}, H_{i}\right), \leqslant k_{i}}^{(n)}(z)+\nu_{\left(f^{t}, H_{i}\right), \leqslant k_{i}}^{(n)}(z)-n \nu_{\left(f, H_{i}\right), \leqslant k_{i}}^{(1)}(z) . \\
\text { and hence } & \sum_{1 \leqslant s<t \leqslant 3} \nu_{P_{s t}^{j l}}(z) \geqslant 2 \sum_{u=1}^{3} \nu_{\left(f^{u}, H_{i}\right), \leqslant k_{i}}^{(n)}(z)-3 n \nu_{\left(f, H_{i}\right), \leqslant k_{i}}^{(1)}(z) .
\end{aligned}
$$

Combining this inequality and the above claim, we have

$$
\sum_{1 \leqslant s<t \leqslant 3} \nu_{P_{s t}^{j l}}(z) \geqslant \sum_{i=j, l}\left(2 \sum_{u=1}^{3} \nu_{\left(f^{u}, H_{i}\right), \leqslant k_{i}}^{(n)}(z)-3 n \nu_{\left(f, H_{i}\right), \leqslant k_{i}}^{(1)}(z)\right)+\sum_{i \neq j, l}\left(4 \nu_{\left(f, H_{i}\right), \leqslant k_{i}}^{(1)}(z)-\chi_{\nu_{i}}(z)\right) .
$$

This yields that

$$
\begin{aligned}
\sum_{1 \leqslant s<t \leqslant 3} N_{P_{s t}^{j l}}(z) \geqslant & \sum_{i=j, l}\left(2 \sum_{u=1}^{3} N_{\left(f^{u}, H_{i}\right), \leqslant k_{i}}^{(n)}(r)-3 n N_{\left(f, H_{i}\right), \leqslant k_{i}}^{(1)}(r)\right) \\
& +\sum_{i \neq j, l}\left(4 N_{\left(f, H_{i}\right), \leqslant k_{i}}^{(1)}(r)-N\left(r, \nu_{i}\right)\right) .
\end{aligned}
$$

On the other hand, be Jensen formula, we easily see that

$$
N_{P_{s t}^{j l}}(z) \leqslant T_{f^{s}}(r)+T_{f^{t}}(r)+o(T(r))(1 \leqslant s<t \leqslant 3) .
$$

Then the inequality (3.16) implies that

$$
2 T(r) \geqslant \sum_{i=j, l}\left(2 \sum_{u=1}^{3} N_{\left(f^{u}, H_{i}\right), \leqslant k_{i}}^{(n)}(r)-3 n N_{\left(f, H_{i}\right), \leqslant k_{i}}^{(1)}(r)\right)+\sum_{i \neq j, l}\left(4 N_{\left(f, H_{i}\right), \leqslant k_{i}}^{(1)}(r)-N\left(r, \nu_{i}\right)\right) .
$$

Summing-up both sides of the above inequalities over all pair $(j, l)$, we obtain

$$
\begin{aligned}
2 T(r) \geq & \frac{2}{n+1} \sum_{u=1}^{3} \sum_{i=1}^{2 n+2} N_{\left(f^{u}, H_{i}\right), \leqslant k_{i}}^{(n)}(r)+\frac{n}{3 \times(n+1)} \sum_{u=1}^{3} \sum_{i=1}^{2 n+2} N_{\left(f^{u}, H_{i}\right), \leqslant k_{i}}^{(1)}(r) \\
& -\frac{n}{n+1} \sum_{i=1}^{2 n+2} N\left(r, \nu_{i}\right)+o(T(r)) .
\end{aligned}
$$


Thus

$$
\begin{aligned}
\sum_{i=1}^{2 n+2} N\left(r, \nu_{i}\right) \geq & \frac{2}{n} \sum_{u=1}^{3} \sum_{i=1}^{2 n+2} N_{\left(f^{u}, H_{i}\right), \leqslant k_{i}}^{(n)}(r)+\frac{1}{3} \sum_{u=1}^{3} \sum_{i=1}^{2 n+2} N_{\left(f^{u}, H_{i}\right), \leqslant k_{i}}^{(1)}(r) \\
& -\frac{2(n+1)}{n} T(r)+o(T(r)) .
\end{aligned}
$$

Using this estimate, from (3.14) we have

$$
\begin{aligned}
(2 n+2) T(r) \geqslant & \left(2+\frac{2}{n}\right) \sum_{u=1}^{3} \sum_{t=1}^{2 n+2} N_{\left(f^{u}, H_{t}\right), \leqslant k_{t}}^{(n)}(r)+\frac{n-1}{3} \sum_{u=1}^{3} \sum_{t=1}^{2 n+2} N_{\left(f_{u}, H_{t}\right), \leqslant k_{t}}^{(1)}(r) \\
& -\frac{2(n+1)}{n} T(r)-(n+1) \sum_{u=1}^{3} \sum_{t=1}^{2 n+2} N_{\left(f^{u}, H_{i}\right),>k_{i}}+o(T(r)) . \\
& \geqslant\left(2+\frac{2}{n}+\frac{n-1}{3 n}\right) \sum_{u=1}^{3} \sum_{t=1}^{2 n+2} N_{\left(f^{u}, H_{t}\right), \leqslant k_{t}}^{(n)}(r)-\frac{2(n+1)}{n} T(r) \\
& -(n+1) \sum_{u=1}^{3} \sum_{t=1}^{2 n+2} N_{\left(f^{u}, H_{i}\right),>k_{i}}+o(T(r)) . \\
& \geqslant\left(2+\frac{2}{n}+\frac{n-1}{3 n}\right) \sum_{u=1}^{3} \sum_{t=1}^{2 n+2} N_{\left(f^{u}, H_{t}\right)}^{(n)}(r)-\frac{2(n+1)}{n} T(r) \\
& -\left(3 n+3+\frac{n-1}{3}\right) \sum_{u=1}^{3} \sum_{t=1}^{2 n+2} N_{\left(f^{u}, H_{i}\right),>k_{i}}+o(T(r)) . \\
& \geqslant\left(2+\frac{2}{n}+\frac{n-1}{3 n}\right)(n+1) T(r)-\frac{2(n+1)}{n} T(r) \\
& -\left(3 n+3+\frac{n-1}{3}\right) \sum_{i=1}^{2 n+2} \frac{1}{k_{i}+1} T(r)+o(T(r)) .
\end{aligned}
$$

Letting $r \longrightarrow+\infty$, we get

$$
2 n+2 \geqslant\left(2+\frac{2}{n}+\frac{n-1}{3 n}\right)(n+1)-\frac{2(n+1)}{n}-\left(3 n+3+\frac{n-1}{3}\right) \sum_{i=1}^{2 n+2} \frac{1}{k_{i}+1} .
$$

Thus

$$
\sum_{i=1}^{2 n+2} \frac{1}{k_{i}+1} \geqslant \frac{n^{2}-1}{10 n^{2}+8 n}
$$

This is a contradiction.

Hence the supposition is impossible. Therefore, $\sharp \mathcal{F}\left(f,\left\{H_{i}, k_{i}\right\}_{i=1}^{2 n+2}, 1\right) \leqslant 2$. We complete the proof of the theorem.

Proof of Theorem 1.4. Let $f^{1}, f^{2}, f^{3} \in \mathcal{F}\left(f,\left\{H_{i}, k_{i}\right\}_{i=1}^{2 n+1}, p\right)$. Suppose that $f^{1} \times f^{2} \times f^{3}$ : $\mathbf{C}^{m} \rightarrow \mathbf{P}^{n}(\mathbf{C}) \times \mathbf{P}^{n}(\mathbf{C}) \times \mathbf{P}^{n}(\mathbf{C})$ is linearly nondegenerate, where $\mathbf{P}^{n}(\mathbf{C}) \times \mathbf{P}^{n}(\mathbf{C}) \times \mathbf{P}^{n}(\mathbf{C})$ 
is embedded into $\mathbf{P}^{(n+1)^{3}-1}(\mathbf{C})$ by Seger imbedding. Then for every $s, t, l$ we have

$$
P:=\operatorname{Det}\left(\begin{array}{ccc}
\left(f^{1}, H_{s}\right) & \left(f^{1}, H_{t}\right) & \left(f^{1}, H_{l}\right) \\
\left(f^{2}, H_{s}\right) & \left(f^{2}, H_{t}\right) & \left(f^{2}, H_{l}\right) \\
\left(f^{3}, H_{s}\right) & \left(f^{3}, H_{t}\right) & \left(f^{3}, H_{l}\right)
\end{array}\right) \not \equiv 0 .
$$

By Lemma 2.4 we have

$$
\begin{aligned}
T(r) \geqslant & \sum_{i=s, t, l}\left(N\left(r, \min \left\{\nu_{\left(f^{u}, H_{i}\right), \leqslant k_{i}} ; 1 \leqslant u \leqslant 3\right\}\right)\right. \\
& \left.-N_{\left(f, H_{i}\right), \leqslant k_{i}}^{(1)}(r)\right)+2 \sum_{i=1}^{2 n+1} N_{\left(f, H_{i}\right), \leqslant k_{i}}^{(1)}(r)+o(T(r)),
\end{aligned}
$$

where $T(r)=\sum_{u=1}^{3} T_{f^{u}}(r)$. Summing-up both sides of the above inequality over all $(s, t, l)$, we obtain

$$
\begin{aligned}
& T(r) \geqslant \frac{1}{2 n+1} \sum_{i=1}^{2 n+1}\left(3 N\left(r, \min \left\{\nu_{\left(f^{u}, H_{i}\right), \leqslant k_{i}} ; 1 \leqslant u \leqslant 3\right\}\right)\right. \\
&\left.+(4 n-1) N_{\left(f, H_{i}\right), \leqslant k_{i}}^{(1)}(r)\right)+o(T(r)) .
\end{aligned}
$$

It is easy to see that for positive integers $a, b, c$ with $\min \{a, p\}=\min \{b, p\}=\min \{c, p\}$, we have

$$
3 \min \{a, b, c\}+(4 n-1) \geqslant \frac{4 n-1+3 p}{2 n+p}(\min \{a, n\}+\min \{b, n\}+\min \{c, n\}) .
$$

Hence

$$
\begin{aligned}
3 N\left(r, \min \left\{\nu_{\left(f^{u}, H_{i}\right), \leqslant k_{i}} ; 1 \leqslant u \leqslant 3\right\}\right) & +(4 n-1) N_{\left(f, H_{i}\right) \leqslant k_{i}}^{(1)}(r) \\
& \geqslant \frac{4 n-1+3 p}{2 n+p} \sum_{u=1}^{3} N_{\left(f, H_{i}\right), \leqslant k_{i}}^{(n)}(r), \quad(1 \leqslant i \leqslant 2 n+1) .
\end{aligned}
$$

Therefore, the inequality (3.17) implies that

$$
\begin{aligned}
T(r) & \geqslant \frac{1}{2 n+1} \sum_{i=1}^{2 n+1} \frac{4 n-1+3 p}{2 n+p} \sum_{u=1}^{3} N_{\left(f, H_{i}\right), \leqslant k_{i}}^{(n)}(r)+o(T(r)) \\
& \geqslant \frac{4 n-1+3 p}{(2 n+1)(2 n+p)} \sum_{i=1}^{2 n+1} \sum_{u=1}^{3}\left(N_{\left(f, H_{i}\right)}^{(n)}(r)-N_{\left(f, H_{i}\right),>k_{i}}^{(n)}(r)\right)+o(T(r)) \\
& \geqslant \frac{4 n-1+3 p}{(2 n+1)(2 n+p)}\left(n-\sum_{i=1}^{2 n+1} \frac{n}{k_{i}+1}\right) T(r)+o(T(r)) .
\end{aligned}
$$

Letting $r \longrightarrow+\infty$, we get

$$
\begin{gathered}
1 \geqslant \frac{4 n-1+3 p}{(2 n+1)(2 n+p)}\left(n-\sum_{i=1}^{2 n+1} \frac{n}{k_{i}+1}\right), \\
\text { i.e., } \sum_{i=1}^{2 n+1} \frac{1}{k_{i}+1} \geqslant \frac{n p-3 n-p}{4 n^{2}+3 n p-n} .
\end{gathered}
$$


This is a contradiction.

Hence, the map $f^{1} \times f^{2} \times f^{3}$ is linearly degenerate. The theorem is proved.

\section{REFERENCES}

[1] Z. Chen and Q. Yan, Uniqueness theorem of meromorphic mappings into $\mathbf{P}^{n}(\mathbf{C})$ sharing $2 N+3$ hyperplanes regardless of multiplicities, Internat. J. Math., 20 (2009), 717-726.

[2] H. Fujimoto, Uniqueness problem with truncated multiplicities in value distribution theory, Nagoya Math. J., 152 (1998), 131-152.

[3] R. Nevanlinna, Einige Eideutigkeitssätze in der Theorie der meromorphen Funktionen, Acta. Math., 48 (1926), 367-391.

[4] J. Noguchi and T. Ochiai, Introduction to Geometric Function Theory in Several Complex Variables, Trans. Math. Monogr. 80, Amer. Math. Soc., Providence, Rhode Island, 1990.

[5] S. D. Quang, Unicity of meromorphic mappings sharing few hyperplanes, Ann. Polon. Math., 102 No. 3 (2011), 255-270.

[6] S. D. Quang, A finiteness theorem for meromorphic mappings sharing few hyperplanes, Kodai Math. J., 102 No. 35 (2012), 463-484.

[7] L. Smiley, Geometric conditions for unicity of holomorphic curves, Contemp. Math. 25 (1983), 149154.

[8] Q. Yan and Z. Chen, Degeneracy theorem for meromorphic mappings with truncated multiplicity, Acta Math. Scientia, 31B (2011) 549-560.

Department of Mathematics, Hanoi National University of Education, 136-Xuan Thuy, Cau Giay, Hanoi, Vienam.

E-mail address: quangsd@hnue.edu.vn 